\title{
An Empirical Assessment Of The Financial Attributes Of Firms Listed On The Athens Stock Exchange
}

George Iatridis, (E-mail: giatridis@econ.uth.gr), University of Thessaly, Greece George Blanas, (E-mail: blanas@teilar.gr), T.E.I. of Larissa, Greece

\begin{abstract}
This paper studies the financial characteristics of firms that are listed on the Athens Stock Exchange. The primary focus of the study is to capture the financial attributes of firms that display positive and negative stock returns, and firms that display low and high standard deviation in their stock returns. The study also examines the behaviour of firms that belong to ATHEX as well as other market indices, such as FTSE 20, FTSE International, etc. The study categorises listed firms based on size, growth, profitability, liquidity and leverage, and assesses their financial behaviour and performance. Firms with high profitability and growth, liquidity and dividend payout tend to attract investors' interest and display positive stock returns. Firms with unfavourable financial figures and uncertainty appear to exhibit significant volatility in their stock returns. Firms that are politically and financially visible, either due to size or because they belong to a market index, tend to carefully organise their actions and meet financial analysts' forecasts. Firms distribute high dividends and pay their creditors timely in order to attract investors, satisfy creditors and favourably affect stock market participants. Overall, firms structure their decision-making so as to improve their financial profile and performance, and influence the picture that the stock market has about their managerial abilities and future prospects.
\end{abstract}

\section{$1 \quad$ Introduction}

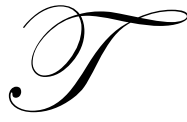

his paper focuses on the period 1 January to 31 December 2004 and examines the financial characteristics of firms that are listed on the Athens Stock Exchange (ASE). The primary focus of the study is to capture the financial attributes of firms that display positive and negative stock returns, and firms that display low and high volatility in their stock returns. The study gives particular emphasis on the identification and interpretation of the financial factors that influence the stock returns of listed firms as well as the volatility that the stock returns appear to exhibit. The paper also examines the behaviour of firms that belong to stock market indices, and mainly whether being a market index constituent affects their decisions and actions. In particular, the study focuses on the main ASE index, i.e. ATHEX, as well as other market indices, such as FTSE 20, FTSE International, etc. To better describe the picture of the Athens stock market, the study categorises the listed firms using accounting measures, such as size, growth, profitability, liquidity and leverage. Here, the study presents the various categorisations of firms that exist in the Athens stock market, and explains how their key financial characteristics influence their financial numbers and actions. The paper shows how the decision to distribute dividends to shareholders impacts on listed firms' financial numbers, while it also presents the distinguishing financial attributes of the latter against firms that pay no dividends. Finally, the paper analyses the financial features of firms that display high and low trading volume, and studies the association between trading volume, stock returns and financial measures.

The motivation of the study is associated with the provision of a thorough financial picture of the Athens Stock market and of the listed firms' behaviour to investors and other market participants, in order to assist them to evaluate firms' financial performance more efficiently and better structure their investment strategies. The study is also motivated by the question of whether the stock returns reflect the true and fair financial picture of firms; that is 
whether the stock market sees through firms' financial performance and rewards those firms with favourable financial figures and prospects. The question that subsequently arises, and is under investigation in the paper, is what kind of firms exhibit positive stock returns and high trading volume. Another set of motives relates to whether listed firms that are politically and financially visible, e.g. those firms that belong to a stock market index, influence their behaviour in order to impress and satisfy market participants and financial authorities, or attract investors.

The remaining sections of the study are as follows. Section 2 presents the literature review of the study. Sections 3 and 4 describe the research hypotheses and the data sets respectively. Section 5 discusses the empirical findings, and Section 6 presents the conclusions of the study.

\section{$2 \quad$ Literature Review}

\subsection{Accounting Choices}

Accounting policy choice is associated with contractual arrangements, such as compensation schemes and debt covenants as well as asset pricing, information asymmetry and political costs (Scott, 1997; Han and Wang, 1998; Francis, 2001; Lambert, 2001). The preparation of financial statements involves accounting policy choice and often requires an exercise of judgement (Jensen and Meckling, 1976; Fama, 1980). Hence, there might be cases where managers' behaviour is opportunistic aiming at the reinforcement of their wealth and interests (Healy, 1985; Dechow and Sloan, 1991; Dye and Verrecchia, 1995).

Managers may use discretionary accounting policies in order to improve the company financial results (Christie and Zimmerman, 1994; Young, 1998; Pope et al, 2000; Bushee, 2001) and positively influence their compensation (Healy, 1985; Dye and Verrecchia, 1995). It follows, thus, that the timing of gains and losses recognition is important (Balsam et al, 1995; Francis, Hanna and Vincent, 1996; Gaver and Gaver, 1998). In certain cases, managers may structure their accounting policy choice so as to transfer earnings from "good" accounting years to "bad" years (DeFond and Park, 1997; Han and Wang, 1998; Guidry et al, 1999). Firms also make use of discretionary accounting policies in order to avoid the risks of bankruptcy, financial distress or debt covenant violation (Holthausen, 1990; Sweeney, 1994; May, 1995). This should be the case especially under significant earnings volatility and price fluctuation (Cahan, 1992; Dechow et al, 1995, 1996 and 1997; Karmon and Lubwama, 1997). ${ }^{1}$

Managers are also inclined to manage the reported accounting numbers in order to influence the behaviour or response of third parties, such as government and tax authorities, regulatory bodies, shareholders, lenders, etc., and avoid the incurrence of agency or political costs (Adiel, 1996; Eldenburg and Soderstrom, 1996; Fields et al, 2001). The political costs are linked to the size of the firm as well as to the size of the reported earnings (Jensen and Murphy, 1990; Ali and Kumar, 1994). Large firms are to a larger extent exposed to political costs, since their large size is likely to attract the attention of government and regulatory bodies, which may in turn assess how consistently the former adhere to accounting regulation (Moses, 1987; Ndubizu and Tsetsekos, 1992). It is reported that firms may manage their key accounting figures in order to be consistent with accounting regulation (Blacconiere et al, 1991; D'Souza, 1998). Following that market participants may not be always able to identify the effects of earnings management (Hirst and Hopkins, 1998), the provision of informative accounting disclosures may capture the impact of accounting policy choice on reported earnings, and thus, mitigate any adverse effects (Blacconiere and Patton, 1994; Elliott and Jacobson, 1994).

\subsection{Stock Returns And Firm Financial Characteristics}

Accounting measures are closely associated with stock returns since they express firms' financial performance (Holthausen and Watts, 2001; Kothari, 2001; Beaver, 2002). Firms tend to manage the reported earnings in order to impress investors and other stock market participants (Moses, 1987; Stulz, 1990; Lewellen, Park and Ro, 1996), or avoid the risk of hostile takeovers (Perry and Williams, 1994; Erickson and Wang, 1999) or any negative stock market response (Burgstahler and Dichev, 1997). It appears that managers tend to influence their decisionmaking and course of action in order to meet financial analysts' forecasts and investors' expectations (Levitt, 1998; Kasznik, 1999; Brown and Caylor, 2005). ${ }^{2}$ In the light of the flexibility that is allowed in financial reporting, the stock 
market seems to value the discretionary treatment of accruals, as it could enhance the information content and explanatory power of the reported earnings (Subramanyam, 1996).

It is evident that financial leverage provides information about the capital structure of the firm and the ability of the latter to efficiently meet its financial obligations and debt covenants (Duke and Hunt, 1990; Press and Weintrop, 1990). Changes in the operating and investing activities of firms tend to significantly affect their financial leverage and capital structure. Thus, changes in financial leverage would tend to reflect changes in firm financial performance (Myers and Majluf, 1984). This implies that changes in financial leverage would also be associated with changes in stock returns, since the latter express firm financial performance and future prospects.

When the share price drops, following for example a bad news announcement, the market value of equity would also tend to drop, leading thus to a higher debt to equity ratio. This is known as the leverage effect (see Merton, 1974; Christie, 1982) and tends to significantly affect investors' perceptions about firms' managerial ability and future prospects. On the other hand, the volatility effect (French, Schwert and Stambaugh, 1987; Campbell and Hentschel, 1992; Bekaert and Wu, 2000; Kim, Morley and Nelson, 2004) would tend to increase investors' required rate of return and affect the share prices accordingly (see also Datar, Naik and Radcliffe, 1998; Easley, Hvidkjaer and O'Hara, 2002). This should also hold when firms borrow funds, in which case investors' required rate of return would tend to be higher following their higher expectations of the efficient use of capital and the future returns that would consequently be generated (Eberhart and Siddique, 2002). Hence, highly leveraged firms would be inclined to make decisions or alter the disclosure of key accounting figures so as to give a positive signal to the stock market and positively affect the stock returns.

Firms are motivated to influence the reported earnings and stock returns, especially when managers' remuneration is linked to stock option schemes and stock performance (Watts and Zimmerman, 1990; Matsunaga, 1995; Yermack, 1997; Chung et al, 2002). To achieve this objective, managers may also provide voluntary disclosures to favourably affect the stock market participants' perceptions and expectations. The literature shows that the abnormal stock returns that follow the stock option awards tend to be significantly positive, while they appear to be insignificant in the period before (Aboody and Kasznick, 2000). Similar considerations would apply in the case where firms are faced with political, agency and regulatory costs, which could potentially affect the stock returns negatively (Watts and Zimmerman, 1986; Blacconiere et al, 1991; Cahan et al, 1997; Doukas et al, 2005). The above costs may be reduced or controlled by monitoring the actions of managers and using financial analysts' forecasts of firm future performance (Nohel and Tarhan, 1998; Lundstrum, 2003). The process of monitoring, however, may be costly and time-consuming, and in certain cases not feasible (Lamont, 1997; Shin and Stulz, 1998; Rajan, Servaes and Zingales, 2000). The literature shows that the use of borrowings may lead to lower agency costs (Jensen, 1986; Jensen, Solberg and Zorn, 1992; Noronha, Shome and Morgan, 1996) since firms will have to meet certain interest payments and debt covenants, while they will be monitored by banks, financial institutions, bond rating agencies, etc. (see also Rozeff, 1982; Alli, Khan and Ramirez, 1993; Dempsey, Laber and Rozeff, 1993; Brous and Kini, 1994).

High firm turnover and profitability would tend to positively affect the stock returns (Brennan and Titman, 1994; Wermers, 2000). The association between turnover and stock returns is also affected by firm liquidity (Lesmond, Ogden and Trzinka, 1999; Rouwenhorst, 1999; Sang-Gyung et al, 2003). A positive relation between the two would suggest that the liquidity of the firm could cover its financial obligations, and thus have a favourable impact on firm value. Such considerations are useful for investors when constructing their portfolios (Domnowitz, Glen and Madhavan, 2001). Firms that operate in a growth area would tend to exhibit positive future prospects, and therefore, the associated stock returns would be expected to be higher reflecting their higher potential (Daniel and Titman, 1997; Dey, 2005). Such firms would like to avoid negative stock market responses to their actions and accounting figures. The literature suggests that they might use forecast management techniques to influence the perceptions of financial analysts and investors (Skinner and Sloan, 2002; Richardson, Teoh and Wysocki, 2004; Bartov et al, 2002; Matsumoto, 2002; Brown and Higgins, 2005). 


\section{$3 \quad$ Research Hypotheses}

\subsection{Stock Returns And Firms' Financial Attributes}

The study focuses on the stock returns of firms that are listed on the ASE, and examines the financial characteristics of firms that exhibit positive returns and firms that display negative returns. ${ }^{3}$ The logistic regression that is employed to identify firms' financial attributes uses a dummy variable as the dependent variable, which is dichotomous and takes two values, i.e. 1 for firms with positive returns, and 0 for firms with negative returns. The formula that is used to obtain the annual stock returns is as follows (Strong, p. 535, 1992):

$$
R_{j t}=\frac{P_{j t}+D_{j t}-P_{j t-1}}{P_{j t-1}},
$$

where $\quad P_{j t} \quad$ is the price of security $j$ at the end of period $t$;

$D_{j t} \quad$ is the dividend paid during period $t$;

$P_{j t-1} \quad$ is the price of security $j$ at the end of period $t-1$.

The hypothesis that is tested is the following:

$\mathbf{H}_{\mathbf{0 1}}$ There is no difference in the financial characteristics between firms that display positive or negative stock returns. The alternative hypothesis is that firms that display positive stock returns have distinctive financial characteristics compared to firms that display negative returns.

\subsection{Volatility In Stock Returns And Firms' Financial Attributes}

The paper also studies the volatility in stock returns and seeks to identify the financial characteristics of firms that display high volatility and firms that exhibit low volatility. The volatility in stock returns is expressed by the standard deviation of stock returns. The logistic regression uses a dummy variable as the dependent variable that takes two values, i.e. 1 for firms with high volatility in stock returns, and 0 for firms with low volatility. This categorisation is performed using the median value of the observations obtained for the variable VOLAT. The hypothesis that is tested is as follows:

$\mathbf{H}_{\mathbf{0} 2}$ There is no difference in the financial characteristics between firms that display high or low volatility in stock returns. The alternative hypothesis is that firms that display high volatility in stock returns have distinctive financial characteristics compared to firms that display low volatility.

\subsection{Factors That Influence The Stock Returns And Their Volatility}

The paper examines the financial measures that influence listed firms' stock returns and their volatility. To carry out the above analysis, the study runs two linear regressions. The first linear regression assesses the association between stock returns and firm financial measures. The second linear regression examines the association between volatility in stock returns and firm financial measures. The hypotheses that are tested are respectively the following:

$\mathbf{H}_{\mathbf{0}}$ Stock returns are not affected by firm financial measures. The alternative hypothesis is that firm financial measures do affect stock returns.

$\mathbf{H}_{\mathbf{0} 3.1}$ The volatility in stock returns is not affected by firm financial measures. The alternative hypothesis is that firm financial measures do affect the volatility in stock returns. 


\subsection{Stock Market Indices And Firms' Financial Attributes}

The study examines the financial behaviour of firms that belong to stock market indices, such as ATHEX, FTSE 20, FTSE International, E-Income and E-IPO. Firstly, the study compares the firms that belong to ATHEX with a control sample, i.e. those firms that are outside ATHEX. The dependent dummy variable in the logistic regression takes two values, i.e. 1 for firms within ATHEX, and 0 for the control sample firms. Secondly, the study examines comparatively the financial characteristics of the firms that belong to the above market indices. The dependent dummy variable in the logistic regression takes the following values: 1 for firms that belong to E-Income, and 0 for firms that belong to ATHEX. Similar logistic regressions are applied for the comparisons between E-IPO vs. ATHEX, FTSE 20 vs. E-Income, and FTSE International vs. E-Income. The hypotheses that are tested are respectively the following:

$\mathbf{H}_{\mathbf{0} 4}$ There is no difference in the financial characteristics between firms that are inside or outside ATHEX. The alternative hypothesis is that firms that belong to ATHEX have distinctive financial characteristics compared to firms that are outside ATHEX.

$\mathbf{H}_{\mathbf{0}}$ 4.1 There is no difference in the financial characteristics between firms that belong to different stock market indices. The alternative hypothesis is that firms that belong to different stock market indices have distinctive financial characteristics.

\subsection{Key Accounting Measures And Firms' Financial Attributes}

Seeking to identify listed firms' financial attributes, the study focuses on key accounting measures, such as firm size, growth, profitability, liquidity and leverage. Firms are categorised into groups based on the size of the above measures, and then tested for differences in their financial behaviour and characteristics. For example, the analysis splits the sample firms into high and low profitability firms, in order to examine their financial attributes and differences (if any). This also encompasses an analysis of the stock return behaviour of the respective groups of firms. The dependent dummy variable in the logistic regression takes the following values: 1 for high profitability firms, and 0 for low profitability firms. This categorisation is performed using the median value of the variable EPS. Similar logistic regressions are applied when using size, growth, liquidity and leverage measures. The hypothesis that is tested is as follows:

$\mathbf{H}_{05}$ There is no difference in the financial characteristics between firms that display high or low size, growth, profitability, liquidity or leverage measures. The alternative hypothesis is that firms that display high size, growth, profitability, liquidity or leverage measures have distinctive financial characteristics compared to firms that display low such measures.

\subsection{Dividend Distribution And Firms' Financial Attributes}

The study examines whether dividend distribution has significant impact on firm financial performance and stock returns. The study splits the sample firms based on whether they distribute dividends or not. The dependent dummy variable in the logistic regression takes the following values: 1 for firms that distribute dividends, and 0 for firms that do not distribute dividends. The hypothesis that is tested is as follows:

$\mathbf{H}_{\mathbf{0}} 6$ There is no difference in the financial characteristics between firms that distribute or do not distribute dividends. The alternative hypothesis is that firms that distribute dividends have distinctive financial characteristics compared to firms that do not distribute dividends.

\subsection{Trading Volume and Firms' Financial Attributes}

The study assesses the association between trading volume and firm financial measures and stock returns, in order to interpret the movements in the trading volume of listed firms. The dependent dummy variable in the logistic regression takes two values, i.e. 1 for firms with positive change in trading volume, and 0 for firms with negative change in trading volume. This categorisation is performed based on the change in trading volume that firms exhibit throughout the period under investigation, and is obtained using the formula below. 
$D T V=\frac{T V_{j t}-T V_{j t-1}}{T V_{j t-1}}$,

where $T V_{j t} \quad$ is the trading volume of security $j$ at the end of period $t$;

$T V_{j t-1} \quad$ is the trading volume of security $j$ at the end of period $t-1$.

The hypothesis that is tested is as follows:

$\mathbf{H}_{\mathbf{0} 7}$ There is no difference in the financial characteristics between firms with positive or negative change in trading volume. The alternative hypothesis is that firms with positive change in trading volume have distinctive financial characteristics compared to firms with negative change in trading volume.

\section{$4 \quad$ Data}

The study has used the binary logistic regression analysis and the Kruskal-Wallis (K-W) test to test the research hypotheses. The empirical analysis concentrates on the accounting period 1 January - 31 December 2004. The sample consists of 254 firms that are listed on the ASE. The study examines the financial characteristics of firms that belong to the Athens stock market as a whole, but also assesses empirically and comparatively the financial attributes of firms that belong to different stock market indices. The study focuses on the following market indices: ATHEX, FTSE 20, FTSE International, E-Income and E-IPO. Financial data regarding firms' stock returns and stock market index structure were obtained from 'www.e-net.gr' and 'www.ase.gr'. ATHEX is a composite price index and is composed of the most highly capitalised shares of the Athens main stock market. ATHEX reflects general trends of the stock market. E-Income consists of firms that display the highest positive change in earnings in the last 3 years. EIPO refers to initial public offerings and consists of firms that listed their shares on the Athens Stock Exchange in the previous 12-month period. FTSE 20 consists of the largest listed firms. The relevant participation criteria relate to capitalisation, marketability and dispersion. FTSE International ${ }^{4}$ is composed of firms that have international exposure, and subsidiaries and interests in foreign countries. Accounting and financial data were collected from firms' financial statements obtained in their physical form. The analysis has excluded banks, insurance, pension and brokerage firms, as their accounting measures are not always comparable with those of industrial firms. Appendix 1 presents the industrial sector structure of the sample firms. Appendix 2 shows the explanatory variables that are employed in the empirical analysis.

\section{$5 \quad$ Empirical Findings}

\subsection{Stock Returns And Firms' Financial Attributes}

Panel A of Table 1 shows that firms that display positive stock returns exhibit significantly different financial characteristics compared to firms that display negative returns. Hence, $H_{O_{l}}$ can be rejected. It appears that the stock market rewards firms that perform well and exhibit good financial performance. In other words, firms that display favourable and positive financial figures have been found to exhibit positive stock returns. In particular, firms with positive stock returns tend to be more profitable (OPM and EPS) and exhibit higher liquidity (CUR) and growth (MVBV) measures. In an efficient stock market, the display of accounting figures, such as the ones presented above, would tend to attract investors and positively impact on firms' stock returns. The specific firms also pay higher dividend (DIVSH) to their shareholders, and thus, positively affect the perceptions of the stock market and the price movements of their share.

The finding that firms with positive stock returns exhibit higher leverage (TLSFU and DEBTE), which in turn reflects their higher financial obligations, such as interest payments and debt covenant restrictions, appears not to adversely affect firms' stock returns. This results from the fact that the higher profitability and liquidity of those firms would aid them to efficiently and timely meet their financial obligations. On the other hand, higher leverage means that firms have more capital available to fulfil their business objectives. This in association with the higher growth potential of the specific firms implies that investors' required rate of return would tend to be higher. The significantly 
positive coefficient of sales per share (SALESHA) shows that size and political costs play a significant role and are taken into consideration by stock market participants. Large firms are significantly exposed to scrutiny and political attention, and therefore, tend to influence their decisions and accounting choices in order to minimise any political and agency costs that might otherwise arise and improve their financial profile (Kim and Kross, 1998). Indeed, Panel A shows that firms with positive stock returns tend to be larger (SALESHA). The results of the K-W test (Panel A of Table 2) are similar with those of the logistic regression presented above.

Table 1 Regression Analysis

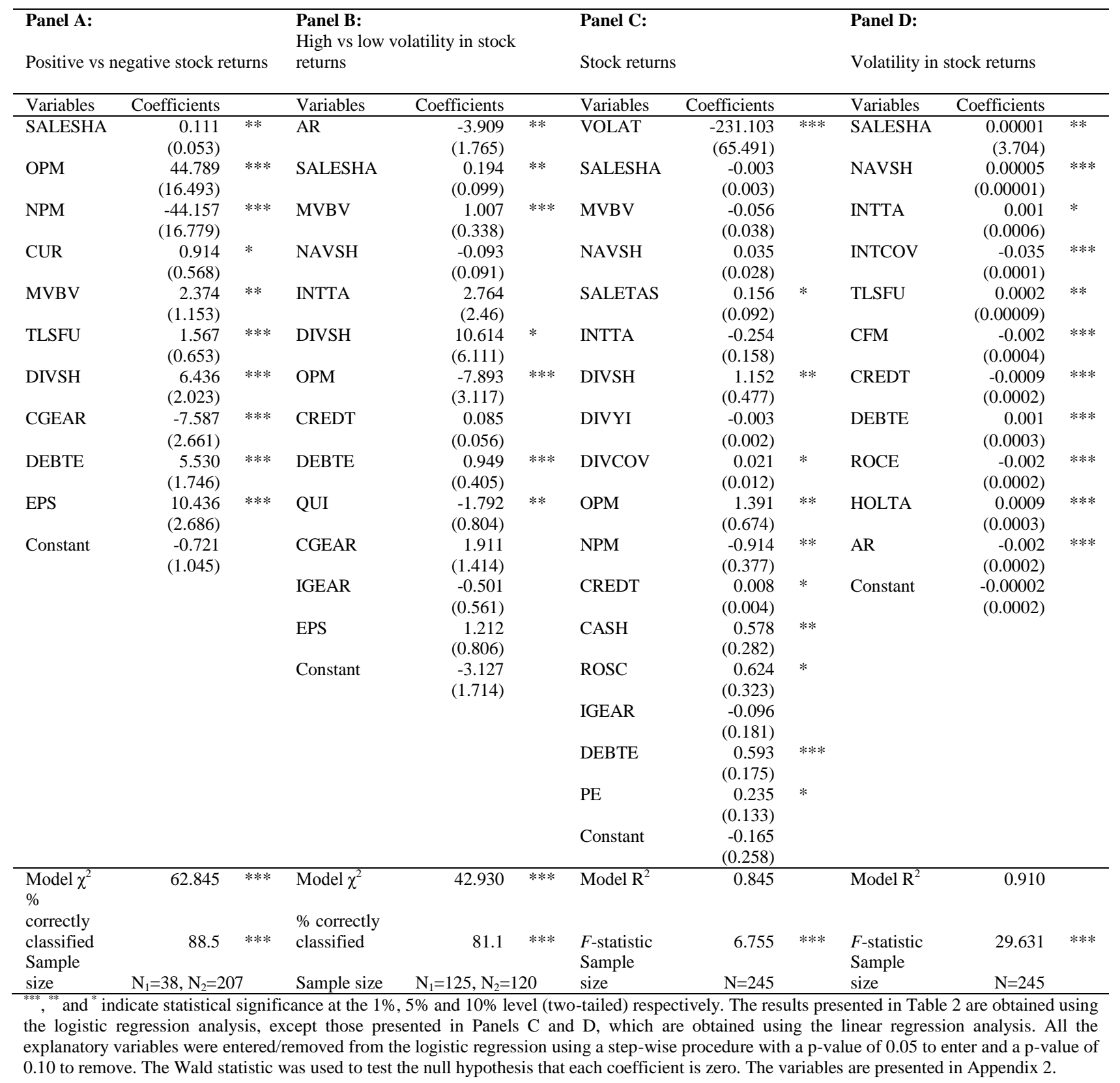


Table 1 Regression Analysis Continued

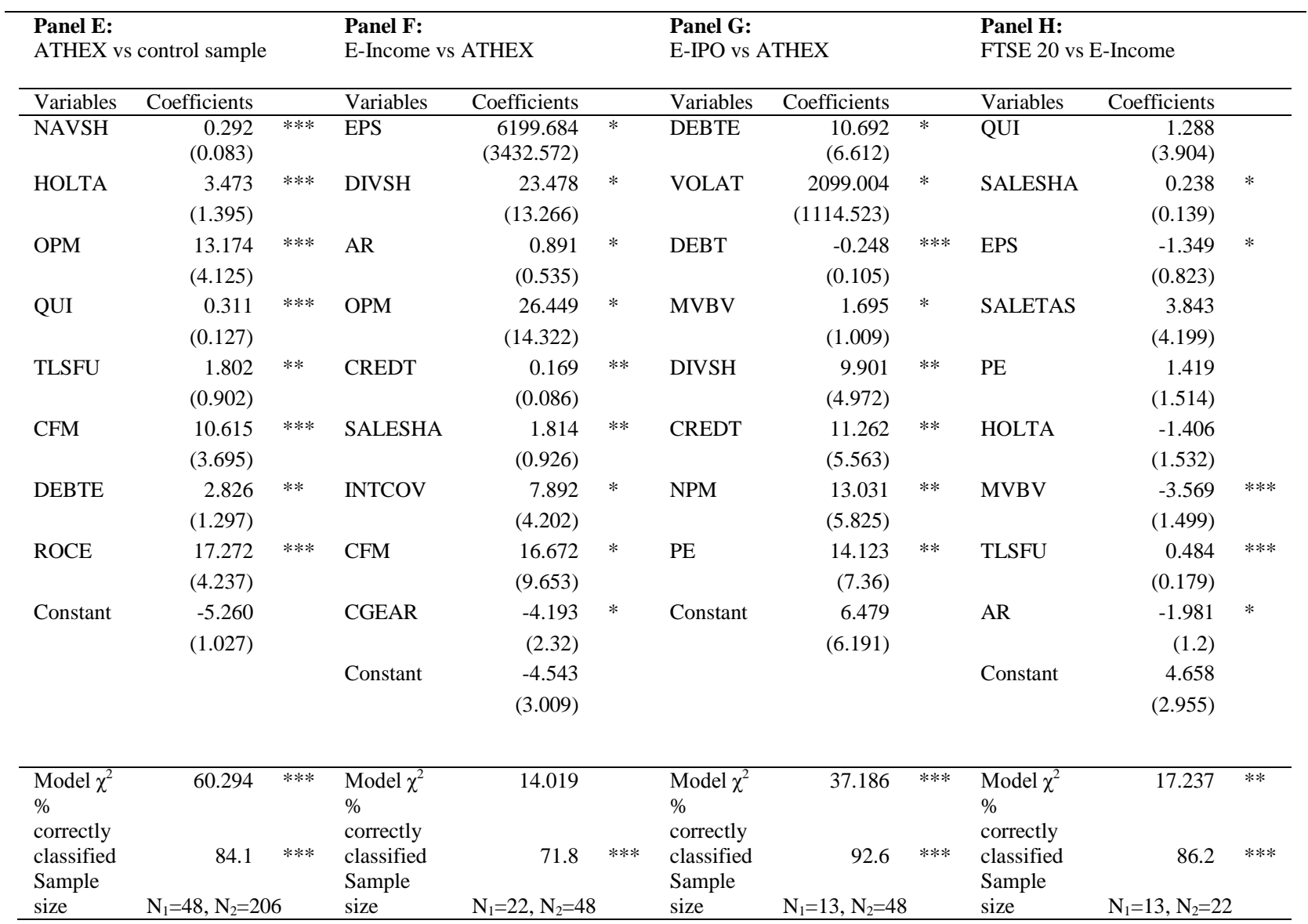


Table 1 Regression Analysis Continued

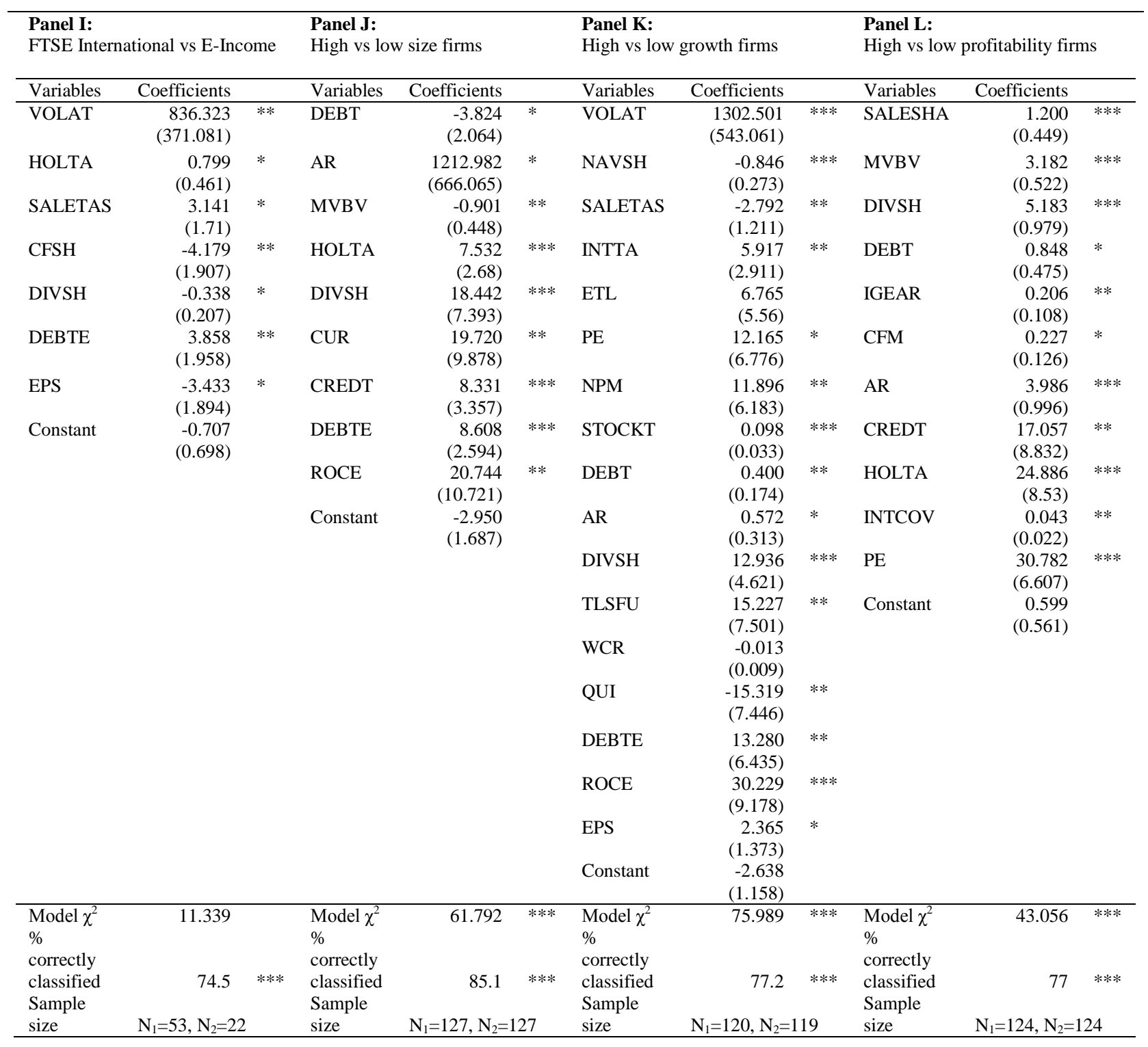


Table 1 Regression Analysis Continued

\begin{tabular}{|c|c|c|c|c|c|c|c|c|c|c|c|}
\hline \multicolumn{3}{|c|}{$\begin{array}{l}\text { Panel M: } \\
\text { High vs low liquidity firms }\end{array}$} & \multicolumn{3}{|c|}{$\begin{array}{l}\text { Panel N: } \\
\text { High vs low leverage firms }\end{array}$} & \multicolumn{3}{|c|}{$\begin{array}{l}\text { Panel O: } \\
\text { Dividend payout vs dividend } \\
\text { retention }\end{array}$} & \multicolumn{3}{|c|}{$\begin{array}{l}\text { Panel P: } \\
\text { Positive vs negative change in trading } \\
\text { volume }\end{array}$} \\
\hline Variables & Coefficients & & Variables & Coefficients & & Variables & Coefficients & & Variables & Coefficients & \\
\hline $\mathrm{AR}$ & $\begin{array}{r}0.611 \\
(0.311)\end{array}$ & $* *$ & SALESHA & $\begin{array}{r}1.270 \\
(0.405)\end{array}$ & *** & NAVSH & $\begin{array}{r}0.948 \\
(0.383)\end{array}$ & **** & $\mathrm{AR}$ & $\begin{array}{r}4.128 \\
(1.665)\end{array}$ & $* * *$ \\
\hline SALETAS & $\begin{array}{r}9.535 \\
(3.914)\end{array}$ & $* * *$ & MVBV & $\begin{array}{r}0.875 \\
(0.332)\end{array}$ & $* * *$ & OPM & $\begin{array}{r}40.712 \\
(15.245)\end{array}$ & $* * *$ & SALESHA & $\begin{array}{r}0.036 \\
(0.023)\end{array}$ & \\
\hline DIVCOV & $\begin{array}{r}5.346 \\
(2.382)\end{array}$ & $* *$ & NAVSH & $\begin{array}{r}0.582 \\
(0.59)\end{array}$ & & NPM & $\begin{array}{r}6.331 \\
(2.952)\end{array}$ & $* *$ & MVBV & $\begin{array}{r}0.757 \\
(0.365)\end{array}$ & $* *$ \\
\hline NPM & $\begin{array}{r}43.788 \\
(24.652)\end{array}$ & $*$ & DIVCOV & $\begin{array}{r}16.546 \\
(7.077)\end{array}$ & $* * *$ & DEBT & $\begin{array}{r}0.493 \\
(0.307)\end{array}$ & $*$ & DIVSH & $\begin{array}{r}7.083 \\
(3.76)\end{array}$ & $*$ \\
\hline STOCKT & $\begin{array}{r}1.436 \\
(0.53)\end{array}$ & $* * *$ & DIVYI & $\begin{array}{r}0.097 \\
(0.036)\end{array}$ & $* * *$ & CFM & $\begin{array}{r}13.447 \\
(7.856)\end{array}$ & $*$ & CREDT & $\begin{array}{r}0.180 \\
(0.099)\end{array}$ & $*$ \\
\hline CREDT & $\begin{array}{r}0.099 \\
(0.044)\end{array}$ & $* *$ & PLOWB & $\begin{array}{r}-0.871 \\
(0.444)\end{array}$ & $* *$ & DEBTE & $\begin{array}{r}2.430 \\
(1.496)\end{array}$ & $*$ & DIVCOV & $\begin{array}{r}0.244 \\
(0.128)\end{array}$ & $* *$ \\
\hline DEBT & $\begin{array}{r}3.116 \\
(1.127)\end{array}$ & $* * *$ & NPM & $\begin{array}{r}-61.779 \\
(20.621)\end{array}$ & $* * *$ & EPS & $\begin{array}{r}9.848 \\
(4.789)\end{array}$ & $* *$ & ROSC & $\begin{array}{r}23.020 \\
(9.056)\end{array}$ & $* * *$ \\
\hline ROSC & $\begin{array}{r}81.580 \\
(37.125)\end{array}$ & $* *$ & STOCKT & $\begin{array}{r}0.063 \\
(0.02)\end{array}$ & $* * *$ & PE & $\begin{array}{r}19.798 \\
(10.003)\end{array}$ & $* *$ & CUR & $\begin{array}{r}1.537 \\
(0.839)\end{array}$ & $*$ \\
\hline CGEAR & $\begin{array}{r}26.598 \\
(9.589)\end{array}$ & $* * *$ & DEBT & $\begin{array}{r}0.057 \\
(0.034)\end{array}$ & $*$ & Constant & $\begin{array}{r}1.008 \\
(1.497)\end{array}$ & & DEBTE & $\begin{array}{r}-0.200 \\
(0.121)\end{array}$ & $*$ \\
\hline INTCOV & $\begin{array}{r}0.974 \\
(0.39)\end{array}$ & $* * *$ & CUR & $\begin{array}{r}0.216 \\
(0.285)\end{array}$ & & & & & $\mathrm{PE}$ & $\begin{array}{r}-44.119 \\
(36.774)\end{array}$ & \\
\hline Constant & $\begin{array}{r}-6.397 \\
(4.537)\end{array}$ & & CFM & $\begin{array}{r}18.270 \\
(10.396)\end{array}$ & $*$ & & & & Constant & $\begin{array}{r}-0.091 \\
(1.388)\end{array}$ & \\
\hline & & & ROCE & $\begin{array}{r}-86.744 \\
(23.432)\end{array}$ & $* * *$ & & & & & & \\
\hline & & & EPS & $\begin{array}{r}-10.881 \\
(4.373)\end{array}$ & $* * *$ & & & & & & \\
\hline & & & $\mathrm{PE}$ & $\begin{array}{r}6.172 \\
(3.208)\end{array}$ & $* *$ & & & & & & \\
\hline & & & Constant & $\begin{array}{r}-4.438 \\
(2.021)\end{array}$ & & & & & & & \\
\hline Model $\chi^{2}$ & 84.002 & $* * *$ & $\underset{\%}{\text { Model } \chi^{2}}$ & 65.745 & $* * *$ & Model $\chi^{2}$ & 123.538 & *** & Model $\chi^{2}$ & 25.678 & **** \\
\hline $\begin{array}{l}\text { correctly } \\
\text { classified } \\
\text { Sample } \\
\text { size } \\
\end{array}$ & $\mathrm{N}_{1}=127, \mathrm{~N}_{2}=1$ & $* * *$ & $\begin{array}{l}\text { correctly } \\
\text { classified }\end{array}$ & 89.3 & $* * *$ & $\begin{array}{l}\% \text { correctly } \\
\text { classified }\end{array}$ & 92.1 & $* * *$ & $\begin{array}{l}\% \text { correctly } \\
\text { classified }\end{array}$ & 84.1 & $* * *$ \\
\hline
\end{tabular}


Table 2 Kruskal - Wallis Statistic

\begin{tabular}{|c|c|c|c|c|c|c|c|c|c|c|c|c|c|c|c|c|c|c|c|c|c|}
\hline \multirow{3}{*}{$\frac{\text { Variables }}{\text { MV }}$} & \multicolumn{3}{|c|}{$\begin{array}{l}\text { Panel A: } \\
\text { Positive vs negative } \\
\text { stock returns }\end{array}$} & \multicolumn{3}{|c|}{$\begin{array}{l}\text { Panel B: } \\
\text { High vs low } \\
\text { volatility in stock } \\
\text { returns }\end{array}$} & \multicolumn{3}{|c|}{$\begin{array}{l}\text { Panel C: } \\
\text { ATHEX vs } \\
\text { control sample }\end{array}$} & \multicolumn{3}{|c|}{$\begin{array}{l}\text { Panel D: } \\
\text { E-Income vs } \\
\text { ATHEX }\end{array}$} & \multicolumn{3}{|c|}{$\begin{array}{l}\text { Panel E: } \\
\text { E-IPO vs } \\
\text { ATHEX }\end{array}$} & \multicolumn{3}{|c|}{$\begin{array}{l}\text { Panel F: } \\
\text { FTSE } 20 \text { vs E- } \\
\text { Income }\end{array}$} & \multicolumn{3}{|c|}{$\begin{array}{l}\text { Panel G: } \\
\text { FTSE } \\
\text { International } \\
\text { vs E-Income }\end{array}$} \\
\hline & \multicolumn{3}{|l|}{ K-W statistic } & \multicolumn{3}{|c|}{$\mathrm{K}-\mathrm{W}$ statistic } & \multicolumn{3}{|c|}{$\mathrm{K}-\mathrm{W}$ statistic } & \multicolumn{3}{|c|}{$\mathrm{K}-\mathrm{W}$ statistic } & \multicolumn{3}{|c|}{$\mathrm{K}-\mathrm{W}$ statistic } & \multicolumn{3}{|c|}{$\mathrm{K}-\mathrm{W}$ statistic } & \multicolumn{3}{|c|}{$\mathrm{K}-\mathrm{W}$ statistic } \\
\hline & 9.838 & $\mathrm{a}$ & & 7.066 & $\mathrm{a}$ & & 93.019 & $\mathrm{a}$ & & 0.074 & $\mathrm{a}$ & & 20.969 & $\mathrm{a}$ & & 7.187 & $\mathrm{a}$ & & 0.130 & $\mathrm{a}$ & \\
\hline $\mathrm{BV}$ & 8.033 & a & $* * *$ & 0.384 & a & & 71.227 & $\mathrm{a}$ & *** & 0.008 & $\mathrm{a}$ & & 14.089 & $\mathrm{a}$ & $* * *$ & 1.820 & b & & 0.054 & a & \\
\hline SALES & 13.339 & a & $* * *$ & 0.011 & $\mathrm{a}$ & & 48.928 & $\mathrm{a}$ & *** & 0.081 & $\mathrm{~b}$ & & 15.150 & a & $* * *$ & 4.269 & a & $* *$ & 0.296 & a & \\
\hline SALESHA & 34.658 & a & $* * *$ & 0.211 & $\mathrm{a}$ & & 20.257 & a & $* * *$ & 0.232 & $\mathrm{~b}$ & & 8.456 & a & $* * *$ & 1.712 & b & & 0.208 & $\mathrm{a}$ & ** \\
\hline NAVSH & 42.699 & a & $* * *$ & 0.227 & a & & 41.157 & a & $* * *$ & 0.090 & $\mathrm{~b}$ & & 15.895 & a & $* * *$ & 0.286 & b & & 0.058 & $\mathrm{~b}$ & \\
\hline SALETAS & 5.926 & a & $* * *$ & 0.193 & a & & 0.033 & a & & 0.289 & $\mathrm{~b}$ & & 0.179 & b & & 0.590 & b & & 0.487 & $\mathrm{a}$ & \\
\hline MVBV & 0.981 & a & $*$ & 16.172 & a & $* * *$ & 2.096 & a & & 0.075 & a & & 2.608 & b & & 4.852 & b & $* *$ & 0.001 & $\mathrm{a}$ & \\
\hline OPM & 12.698 & a & $* * *$ & 0.946 & $\mathrm{a}$ & & 24.730 & a & **** & 0.121 & $\mathrm{~b}$ & & 3.291 & a & $*$ & 0.066 & b & & 0.077 & $\mathrm{a}$ & \\
\hline NPM & 11.709 & a & $* * *$ & 1.208 & $\mathrm{a}$ & & 19.505 & a & *** & 0.074 & $\mathrm{a}$ & * & 0.150 & a & & 0.168 & b & & 0.062 & a & \\
\hline ROSC & 11.052 & a & $* * *$ & 0.472 & $\mathrm{a}$ & & 11.941 & a & **** & 0.044 & b & & 0.052 & b & & 3.215 & b & * & 0.099 & $\mathrm{a}$ & \\
\hline ROCE & 10.360 & $\mathrm{a}$ & $* * *$ & 0.390 & $\mathrm{a}$ & & 6.745 & a & *** & 0.085 & $\mathrm{~b}$ & & 0.004 & b & & 1.157 & b & & 0.319 & $\mathrm{a}$ & \\
\hline EPS & 33.178 & a & $* * *$ & 1.149 & $\mathrm{a}$ & & 33.211 & a & *** & 0.035 & b & & 6.462 & a & $* * *$ & 3.196 & b & $*$ & 0.036 & $\mathrm{~b}$ & ** \\
\hline STOCKT & 2.401 & a & & 0.001 & $\mathrm{a}$ & & 11.482 & a & *** & 0.529 & $\mathrm{a}$ & & 6.962 & a & $* * *$ & 1.074 & b & & 0.653 & $\mathrm{~b}$ & \\
\hline CREDT & 2.469 & a & & 0.030 & b & & 0.495 & $\mathrm{a}$ & & 0.057 & $\mathrm{~b}$ & & 0.086 & b & & 0.063 & b & & 0.053 & a & \\
\hline DEBT & 9.972 & a & $* * *$ & 0.020 & b & & 14.050 & a & *** & 0.237 & $\mathrm{a}$ & & 4.693 & a & $* *$ & 3.464 & b & & 0.031 & $\mathrm{~b}$ & \\
\hline CUR & 0.111 & a & & 0.607 & $\mathrm{a}$ & & 0.844 & a & & 0.289 & $\mathrm{~b}$ & & 0.194 & b & & 0.213 & a & & 0.933 & $\mathrm{a}$ & \\
\hline $\mathrm{CASH}$ & 4.644 & a & $* *$ & 0.011 & b & & 3.292 & a & * & 0.025 & b & & 1.434 & a & & 0.245 & b & & 0.092 & a & \\
\hline QUI & 0.010 & b & & 0.718 & $\mathrm{a}$ & & 2.766 & a & $*$ & 0.014 & $\mathrm{~b}$ & & 0.025 & a & & 0.007 & a & & 0.425 & $\mathrm{a}$ & \\
\hline CFM & 4.101 & a & $* *$ & 2.998 & b & $*$ & 20.898 & a & *** & 0.044 & b & & 2.086 & a & & 0.066 & a & & 0.001 & $\mathrm{~b}$ & \\
\hline CFSH & 45.544 & a & $* * *$ & 2.001 & $\mathrm{a}$ & & 35.534 & a & **** & 0.001 & a & & 9.666 & a & $* * *$ & 2.228 & b & & 0.137 & $\mathrm{~b}$ & \\
\hline TLSFU & 0.016 & b & & 3.317 & a & $*$ & 1.260 & $\mathrm{~b}$ & & 0.548 & $\mathrm{a}$ & & 2.060 & b & & 1.470 & b & & 0.395 & b & \\
\hline CGEAR & 0.008 & a & & 3.769 & $\mathrm{a}$ & $* *$ & 0.480 & $\mathrm{~b}$ & & 1.063 & a & & 1.768 & b & & 3.338 & $\mathrm{a}$ & $*$ & 0.824 & $\mathrm{~b}$ & \\
\hline INTCOV & 16.574 & a & $* * *$ & 2.515 & a & & 15.839 & a & $* * *$ & 1.527 & b & & 2.814 & a & $*$ & 4.332 & a & & 0.216 & a & \\
\hline IGEAR & 2.476 & b & & 4.063 & b & & 2.123 & $\mathrm{~b}$ & & 1.436 & a & & 1.633 & b & & 0.026 & b & & 1.108 & $\mathrm{~b}$ & \\
\hline DEBTE & 0.504 & a & & 1.353 & b & & 4.636 & a & $* *$ & 2.271 & a & & 0.173 & a & & 3.130 & a & $*$ & 1.968 & $\mathrm{a}$ & $*$ \\
\hline INTTA & 0.611 & a & & 2.061 & a & & 3.032 & $\mathrm{a}$ & & 0.000 & a & & 0.322 & b & & 1.526 & b & & 0.004 & a & \\
\hline HOLTA & 0.141 & a & & 0.285 & a & & 3.123 & $\mathrm{a}$ & * & 0.796 & b & & 7.167 & a & & 0.590 & a & & 0.066 & $\mathrm{a}$ & \\
\hline AR & & & & 28.802 & b & $* * *$ & 4.898 & $\mathrm{a}$ & $* *$ & 1.637 & a & $*$ & 0.076 & a & & 0.820 & b & $*$ & 1.818 & a & \\
\hline VOLAT & 0.770 & a & & & & & 29.907 & a & & 0.914 & a & & 17.235 & a & $* * *$ & 2.571 & b & & 1.542 & $\mathrm{a}$ & ** \\
\hline DIVSH & 19.876 & a & $* * *$ & 2.156 & a & & 23.556 & a & **** & 1.315 & b & & 11.490 & a & $* * *$ & 0.919 & b & & 0.844 & $\mathrm{a}$ & \\
\hline DIVYI & 5.080 & a & $* *$ & 0.023 & a & & 6.605 & a & $* * *$ & 4.761 & a & $* *$ & 5.811 & a & $* *$ & 1.665 & a & & 4.767 & $\mathrm{a}$ & \\
\hline DIVCOV & 6.297 & a & $* * *$ & 0.839 & a & & 0.287 & a & & 0.018 & a & & 1.288 & b & & 1.191 & b & & 0.436 & $\mathrm{~b}$ & \\
\hline PLOWB & 0.033 & a & & 0.645 & a & & 0.057 & $\mathrm{~b}$ & & 0.169 & a & & 6.855 & b & & 1.685 & b & & 0.440 & $\mathrm{~b}$ & \\
\hline $\mathrm{PE}$ & 2.809 & $\mathrm{~b}$ & & 0.300 & b & & 0.563 & $\mathrm{a}$ & & 0.557 & a & & 0.005 & a & & 1.184 & a & & 0.002 & $\mathrm{~b}$ & \\
\hline
\end{tabular}
$\mathrm{PE}$

, and "indicate statistical significance at the $1 \%, 5 \%$ and $10 \%$ level respectively. ${ }^{\text {a }}$ indicates that the mean rank of the KruskalWallis (K-W) statistic is larger for: Panel A: firms with high stock returns; Panel B: firms with high volatility in stock returns; Panel C: firms that belong to ATHEX; Panel D: firms that belong to E-Income; Panel E: firms that belong to E-IPO; Panel F: firms that belong to FTSE 20; Panel G: firms that belong to FTSE International; Panel H: large firms; Panel I: high growth firms; Panel J: high profitability firms; Panel K: high liquidity firms; Panel L: high leverage firms; Panel M: firms that pay dividends; Panel N: firms with positive change in trading volume. In Panel A, the variable "stock returns" (AR) is not included in the analysis because it is used to categorise the sample firms into firms with positive and negative returns. In Panel B, the variable "volatility in stock returns" (VOLAT) is not included in the analysis because it is used to categorise the sample firms into firms with high and low volatility in stock returns. In Panel $\mathrm{H}$, the size variables are not included in the analysis because the sample firms have been categorised based on size into large and small firms. In Panel I, the growth variable "market value to book value" (MVBV) is not included in the analysis because it is used to categorise the sample firms into high and low growth firms. In Panel J, the profitability variables are not included in the analysis because the sample firms have been categorised based on profitability into high and low profitability firms. In Panel $\mathrm{K}$, the liquidity variables are not included in the analysis because the sample firms have been categorized based on liquidity into high and low liquidity firms. In Panel L, the leverage variables are not included in the analysis because the sample firms have been categorised based on leverage into high and low leverage firms. In Panel M, the dividend variables are not included in the analysis because the sample firms have been categorised based on dividend distribution into firms that pay dividends and firms that do not distribute dividends. The variables are presented in Appendix 2. 
Table 2 Kruskal - Wallis Statistic (cntd.)

\begin{tabular}{|c|c|c|c|c|c|c|c|c|c|c|c|c|c|c|c|c|c|c|c|c|c|}
\hline \multirow{4}{*}{$\frac{\text { Variables }}{\text { MV }}$} & \multicolumn{3}{|c|}{$\begin{array}{l}\text { Panel H: } \\
\text { High vs low size } \\
\text { firms }\end{array}$} & \multicolumn{3}{|c|}{$\begin{array}{l}\text { Panel I: } \\
\text { High vs low } \\
\text { growth firms }\end{array}$} & \multicolumn{3}{|c|}{$\begin{array}{l}\text { Panel J: } \\
\text { High vs low } \\
\text { profitability } \\
\text { firms }\end{array}$} & \multicolumn{3}{|c|}{$\begin{array}{l}\text { Panel K: } \\
\text { High vs low } \\
\text { liquidity firms }\end{array}$} & \multicolumn{3}{|c|}{$\begin{array}{l}\text { Panel L: } \\
\text { High vs low } \\
\text { leverage firms }\end{array}$} & \multicolumn{3}{|c|}{$\begin{array}{l}\text { Panel M: } \\
\text { Dividend payout } \\
\text { vs dividend } \\
\text { retention }\end{array}$} & \multicolumn{3}{|c|}{$\begin{array}{l}\text { Panel N: } \\
\text { Positive vs } \\
\text { negative } \\
\text { change in } \\
\text { trading volume }\end{array}$} \\
\hline & \multicolumn{3}{|c|}{ K-W } & \multicolumn{3}{|c|}{$\mathrm{K}-\mathrm{W}$} & \multicolumn{3}{|c|}{$\mathrm{K}-\mathrm{W}$} & \multicolumn{3}{|c|}{ K-W } & \multicolumn{3}{|c|}{ K-W } & \multicolumn{3}{|c|}{ K-W } & \multicolumn{3}{|c|}{$\mathrm{K}-\mathrm{W}$} \\
\hline & statistic & & & statistic & & & statistic & & & statistic & & & statistic & & & statistic & & & statistic & & \\
\hline & & & & 4.114 & $\mathrm{a}$ & & 17.612 & $\mathrm{a}$ & & 0.013 & $\mathrm{~b}$ & & 13.349 & $\mathrm{a}$ & & 12.242 & $\mathrm{a}$ & & 0.810 & & \\
\hline BV & & & & 33.097 & $\mathrm{~b}$ & $* * *$ & 7.535 & a & $* * *$ & 0.186 & $\mathrm{~b}$ & & 12.627 & a & $* * *$ & 1.546 & $\mathrm{a}$ & & 1.215 & $\mathrm{~b}$ & \\
\hline SALES & & & & 3.780 & $\mathrm{~b}$ & $* *$ & 22.756 & a & *** & 8.726 & $\mathrm{a}$ & $* * *$ & 24.187 & $\mathrm{a}$ & $* * *$ & 7.530 & a & $* * *$ & 3.765 & $\mathrm{a}$ & $* *$ \\
\hline SALESHA & & & & 0.516 & $\mathrm{~b}$ & & 70.281 & $\mathrm{a}$ & $* * *$ & 2.447 & $\mathrm{~b}$ & & 8.844 & $\mathrm{a}$ & $* * *$ & 9.013 & $\mathrm{a}$ & $* * *$ & 1.357 & $\mathrm{a}$ & \\
\hline NAVSH & & & & 5.374 & $\mathrm{~b}$ & $* *$ & 73.757 & a & *** & 1.308 & a & & 1.376 & a & & 9.027 & a & $* * *$ & 9.632 & a & *** \\
\hline SALETAS & & & & 6.379 & a & $* * *$ & 14.769 & $\mathrm{a}$ & $* * *$ & 3.775 & $\mathrm{~b}$ & & 0.120 & $\mathrm{~b}$ & & 4.802 & $\mathrm{a}$ & $* *$ & 1.695 & $\mathrm{~b}$ & \\
\hline MVBV & 3.368 & a & & & & & 8.541 & a & $* * *$ & 0.374 & $\mathrm{a}$ & & 2.468 & $\mathrm{~b}$ & & 5.825 & $\mathrm{a}$ & $* *$ & 6.096 & a & $* * *$ \\
\hline OPM & 12.831 & $\mathrm{a}$ & $* * *$ & 8.340 & $\mathrm{a}$ & $* * *$ & & & & 15.395 & $\mathrm{a}$ & $* * *$ & 1.019 & $\mathrm{~b}$ & & 50.270 & $\mathrm{a}$ & $* * *$ & 2.009 & a & \\
\hline NPM & 7.522 & $\mathrm{a}$ & *** & 8.008 & $\mathrm{a}$ & $* * *$ & & & & 18.086 & $\mathrm{a}$ & $* * *$ & 1.235 & b & & 60.290 & $\mathrm{a}$ & $* * *$ & 3.737 & $\mathrm{a}$ & $* *$ \\
\hline ROSC & 2.064 & a & & 13.403 & $\mathrm{a}$ & *** & & & & 3.919 & $\mathrm{a}$ & $* *$ & 0.067 & $\mathrm{a}$ & & 52.048 & $\mathrm{a}$ & $* * *$ & 1.006 & $\mathrm{a}$ & \\
\hline ROCE & 0.164 & $\mathrm{a}$ & & 23.688 & $\mathrm{a}$ & *** & & & & 0.557 & $\mathrm{~b}$ & & 0.013 & $\mathrm{a}$ & & 22.201 & $\mathrm{a}$ & $* * *$ & 0.694 & $\mathrm{a}$ & \\
\hline EPS & 12.569 & a & $* * *$ & 4.170 & $\mathrm{a}$ & $* *$ & & & & 5.575 & $\mathrm{a}$ & $* *$ & 0.290 & $\mathrm{a}$ & & 65.634 & $\mathrm{a}$ & $* * *$ & 6.149 & a & $* * *$ \\
\hline STOCKT & 10.766 & $\mathrm{a}$ & *** & 3.587 & $\mathrm{a}$ & $*$ & 4.434 & $\mathrm{a}$ & $* *$ & & & & 0.583 & $\mathrm{a}$ & & 0.047 & $\mathrm{~b}$ & & 4.518 & $\mathrm{~b}$ & $* *$ \\
\hline CREDT & 1.396 & $\mathrm{a}$ & & 0.087 & a & & 3.269 & a & $*$ & & & & 0.420 & $\mathrm{~b}$ & & 4.289 & $\mathrm{a}$ & ** & 0.002 & a & \\
\hline DEBT & 6.687 & a & $* * *$ & 2.388 & a & & 15.616 & a & *** & & & & 4.467 & $\mathrm{a}$ & $* *$ & 0.944 & $\mathrm{a}$ & & 2.067 & $\mathrm{~b}$ & \\
\hline CUR & 0.002 & a & & 0.439 & a & & 4.438 & a & $* *$ & & & & 3.033 & $\mathrm{~b}$ & $*$ & 8.598 & $\mathrm{a}$ & $* * *$ & 2.651 & $\mathrm{a}$ & * \\
\hline CASH & 2.103 & $\mathrm{a}$ & & 2.796 & a & * & 16.104 & $\mathrm{a}$ & *** & & & & 5.234 & $\mathrm{~b}$ & $* *$ & 5.800 & $\mathrm{a}$ & $* *$ & 4.927 & $\mathrm{a}$ & $* *$ \\
\hline QUI & 0.037 & a & & 0.433 & $\mathrm{a}$ & & 4.069 & a & $* *$ & & & & 3.807 & $\mathrm{~b}$ & $* *$ & 4.259 & $\mathrm{a}$ & $* *$ & 2.656 & a & $*$ \\
\hline CFM & 11.872 & $\mathrm{a}$ & *** & 6.325 & $\mathrm{a}$ & *** & 45.597 & $\mathrm{a}$ & *** & & & & 2.040 & $\mathrm{~b}$ & & 27.266 & $\mathrm{a}$ & $* * *$ & 4.979 & a & $* *$ \\
\hline CFSH & 11.651 & $\mathrm{a}$ & $* * *$ & 0.939 & a & & \#\#\#\# & a & *** & & & & 4.387 & a & $* *$ & 28.469 & $\mathrm{a}$ & $* * *$ & 6.582 & a & $* * *$ \\
\hline TLSFU & 1.290 & $\mathrm{~b}$ & & 0.762 & $\mathrm{a}$ & & 1.180 & $\mathrm{~b}$ & & 48.331 & $\mathrm{~b}$ & $* * *$ & & & & 3.119 & $\mathrm{a}$ & $*$ & 3.951 & $\mathrm{~b}$ & $* *$ \\
\hline CGEAR & 0.359 & $\mathrm{~b}$ & & 0.028 & $\mathrm{~b}$ & & 1.542 & $\mathrm{~b}$ & & 35.619 & $\mathrm{~b}$ & $* * *$ & & & & 6.236 & $\mathrm{~b}$ & $* * *$ & 4.516 & $\mathrm{~b}$ & $* *$ \\
\hline INTCOV & 8.683 & a & *** & 8.069 & a & & 80.532 & a & *** & 12.711 & $\mathrm{a}$ & $* * *$ & & & & 48.942 & $\mathrm{a}$ & $* * *$ & 0.406 & a & \\
\hline IGEAR & 4.683 & $\mathrm{~b}$ & $* *$ & 2.475 & $\mathrm{a}$ & * & 6.344 & $\mathrm{a}$ & $* * *$ & 5.055 & $\mathrm{~b}$ & $* *$ & & & & 2.038 & $\mathrm{a}$ & & 1.660 & $\mathrm{~b}$ & \\
\hline DEBTE & 6.553 & a & *** & 5.139 & $\mathrm{a}$ & $* *$ & 0.282 & $\mathrm{a}$ & & 2.648 & $\mathrm{~b}$ & $*$ & & & & 0.999 & $\mathrm{~b}$ & & 7.276 & $\mathrm{~b}$ & $* * *$ \\
\hline INTTA & 0.097 & $\mathrm{a}$ & & 2.089 & $\mathrm{a}$ & & 0.207 & $\mathrm{a}$ & & 0.318 & $\mathrm{a}$ & & 0.093 & $\mathrm{~b}$ & & 0.848 & $\mathrm{a}$ & & 2.890 & a & \\
\hline HOLTA & 17.940 & a & **** & 4.755 & $\mathrm{~b}$ & & 0.231 & $\mathrm{~b}$ & & 7.840 & $\mathrm{~b}$ & & 3.246 & $\mathrm{a}$ & & 1.307 & $\mathrm{a}$ & & 1.550 & $\mathrm{~b}$ & \\
\hline $\mathrm{AR}$ & 15.586 & $\mathrm{a}$ & *** & 0.043 & $\mathrm{~b}$ & & 52.710 & $\mathrm{a}$ & *** & 0.395 & $\mathrm{a}$ & & 1.399 & $\mathrm{a}$ & & 10.123 & a & $* * *$ & 3.384 & $\mathrm{a}$ & * \\
\hline VOLAT & 1.585 & a & & 24.989 & $\mathrm{a}$ & $* * *$ & 3.195 & $\mathrm{a}$ & & 0.463 & $\mathrm{a}$ & & 0.031 & $\mathrm{~b}$ & & 0.890 & a & & 3.099 & a & \\
\hline DIVSH & 19.454 & $\mathrm{a}$ & *** & 4.619 & $\mathrm{a}$ & $* *$ & \#\#\#\# & $\mathrm{a}$ & $* * *$ & 6.957 & $\mathrm{a}$ & $* * *$ & 0.003 & $\mathrm{a}$ & & & & & 1.157 & a & * \\
\hline DIVYI & 7.023 & a & *** & 0.019 & $\mathrm{a}$ & & 63.575 & $\mathrm{a}$ & $* * *$ & 6.622 & $\mathrm{a}$ & $* * *$ & 0.085 & $\mathrm{a}$ & & & & & 0.815 & b & \\
\hline DIVCOV & 1.306 & $\mathrm{~b}$ & & 1.269 & $\mathrm{a}$ & & 25.448 & $\mathrm{a}$ & *** & 1.008 & $\mathrm{~b}$ & & 0.073 & $\mathrm{a}$ & & & & & 1.810 & a & \\
\hline PLOWB & 0.828 & $\mathrm{~b}$ & & 0.020 & $\mathrm{a}$ & & 3.248 & $\mathrm{a}$ & & 0.583 & $\mathrm{~b}$ & & 1.059 & $\mathrm{a}$ & & 27.544 & $\mathrm{~b}$ & & 0.289 & $\mathrm{~b}$ & \\
\hline $\mathrm{PE}$ & 2.601 & $\mathrm{a}$ & & 0.702 & a & & 2.141 & $\mathrm{~b}$ & & 6.542 & $\mathrm{a}$ & $* * *$ & 0.521 & $\mathrm{~b}$ & & 42.652 & $\mathrm{a}$ & $* * *$ & 1.232 & b & \\
\hline
\end{tabular}

\subsection{Volatility In Stock Returns And Firms' Financial Attributes}

Panel B of Table 1 shows that there are significant financial differences between firms that display high and low volatility in stock returns. ${ }^{5}$ Thus, $H_{02}$ can be rejected. It appears that stock volatility is closely related to firm financial performance and investors' perceptions and expectations. Panel B shows that firms that display high volatility in stock returns tend to exhibit higher leverage (DEBTE), which in turn adversely affects firm profitability (OPM) and liquidity (QUI) measures. The specific firms also display higher growth potential (MVBV), which signifies that they may currently use more debt finance, in order to realise their business plans, expecting to generate higher returns in future periods. Lower profitability and liquidity in combination with higher leverage and uncertainty about firms' future potential would give a negative signal to investors about firms' current financial performance and future potential. Consequently, this would tend to have a negative impact on firms' stock returns. Indeed, Panel B shows that firms with high stock volatility experience negative stock returns (AR). ${ }^{6}$ Given their high stock return volatility and despite their lower profitability, the specific firms appear to distribute higher dividend payout (DIVSH) in order to attract investors and improve their market profile. The fact that firms with high stock volatility are larger (SALESHA) shows that large firms are subjected to public scrutiny and examination, which means that their stock return is more volatile and sensitive to investors' perceptions and understanding of managers' actions. In a similar 
vein, the K-W test (Panel B of Table 2) shows that firms with high stock return volatility display lower stock returns and liquidity, and higher size, growth and leverage measures.

\subsubsection{Factors That Influence The Stock Returns}

Panel $\mathrm{C}$ of Table 1 shows that firm financial measures do affect stock returns. Therefore, $H_{03}$ can be rejected. The behaviour of stock returns is closely associated with firm financial numbers and performance. Investors tend to favour stable stock returns (Gordon, 1964). This implies that unstable and volatile stock returns might adversely impact on investors' perceptions. The size of the market reaction to stock return volatility would of course depend upon investors' investment preferences. Indeed, Panel C shows that volatility in stock returns (VOLAT) appears to adversely affect firms' stock returns.

The results show that the stock market appears to appreciate favourable firm financial figures, and tends to reward firms accordingly. In particular, size (SALETAS), profitability (OPM and ROSC) and liquidity (CASH) appear to positively influence the stock returns. Also, the distribution of dividends to shareholders (DIVSH and DIVCOV) has a positive impact on stock returns. The price to earnings ratio (PE), which reflects firms' future prospects and potential, is positively valued by market participants, and positively affects the stock returns. It should be stressed that when assessing a firm's financial performance, PE should be carefully examined, because in certain cases, it may be overvalued. Therefore, it should be set against the average figure of the sector or the market. Panel C shows that the stock returns are positively affected by signs of positive managerial performance. In particular, paying creditors (CREDT) timely gives a positive sign to the stock market about firms' credibility, and at the same time enhances firms' creditability. The finding that high leverage (DEBTE) affects the stock returns positively signifies that firms would have more capital available to support their investment plans and reinforce their growth potential. In the specific set of results, it appears that the high leverage does not adversely affect firms' liquidity, profitability or stock returns.

\subsubsection{Factors That Influence The Volatility In Stock Returns}

Panel D of Table 1 shows that the volatility in stock returns is significantly affected by firm financial measures. Thus, $H_{03.1}$ can be rejected. The volatility in stock returns expresses the sensitivity of stock returns to firm financial performance and uncertainty. Assuming that large firms attract more political and regulatory attention than small firms, the actions and financial results of the former would tend to affect investors' perceptions and have a more sound impact on stock returns (Zimmerman, 1983). This would therefore affect the behaviour of stock returns, which would be expressed in terms of higher or lower volatility depending upon the expectations and preferences of the stock market. Indeed, the results show that the volatility in stock returns is significantly affected by firm size (SALESHA and NAVSH). Similar considerations would apply in the case of intangibles to total assets (INTTA) and holdings to total assets (HOLTA), which carry significantly positive coefficients. It follows that the uncertainty regarding the valuation and carrying value of intangibles, and the benefits or losses that are related to holdings would be expected to influence firms' stock returns, and potentially make them more volatile. Higher leverage measures (TLSFU and DEBTE) in association with lower profitability (ROCE), liquidity (CFM) and interest cover (INTCOV) would certainly lead to volatile stock returns, reflecting investors' worries about firms' financial integrity and future prospects. The considerations above would lead to a situation where firms would be unable or less able to pay back timely and in consistency with the debt agreements. This situation would worsen firms' financial position and further increase the volatility in stock returns. Indeed, Panel D shows that the creditor turnover (CREDT) carries a negative coefficient. Following the findings described above, it can be inferred that high volatility in stock returns would tend to negatively impact on stock returns. The results are consistent with this statement, and show that stock returns (AR) carry a negative coefficient.

\subsubsection{ATHEX And Firms' Financial Attributes}

Panel E of Table 1 shows that constituent firms and non-constituent firms of ATHEX exhibit significantly different financial attributes. Thus, $H_{0_{4}}$ can be rejected. Panel E shows that firms that belong to ATHEX are larger (NAVSH) and exhibit higher holdings to total assets (HOLTA). They also display higher leverage measures (TLSFU 
and DEBTE). Despite the higher leverage and the related financial costs, the specific firms appear to exhibit higher profitability (OPM and ROCE) and liquidity (QUI and CFM). This should give a positive signal to the stock market about the robustness of their profitability and liquidity, and also reinforce their financial position and creditability. Constituent firms of ATHEX would tend to receive attention by market participants and government authorities. It appears, therefore, that since their actions are visible and subjected to scrutiny, they would seek to make careful financial decisions and exhibit a favourable financial picture. The results of the $\mathrm{K}-\mathrm{W}$ test (Panel C of Table 2) are similar with those of the logistic regression presented above, and also show that firms that belong to ATHEX exhibit higher dividend payout and positive stock returns.

\subsubsection{E-Income vs. ATHEX}

Panel $\mathrm{F}$ of Table 1 shows that firms that belong to E-Income exhibit different financial characteristics compared to firms that belong to ATHEX. Thus, $H_{04.1}$ can be rejected. Panel F indicates that constituent firms of EIncome tend to be larger (SALESHA) and display higher liquidity (CFM). They also exhibit higher profitability (EPS and OPM) and distribute higher dividends (DIVSH). Following their higher profitability and liquidity, the specific firms appear to adequately meet their financial obligations. In particular, they display higher creditor turnover (CREDT) and interest cover (INTCOV). Overall, the results show that firms that belong to E-Income tend to exhibit favourable financial numbers, which appear to influence their stock market picture and positively impact on their stock returns (AR). The K-W test (Panel D of Table 2) shows that firms that belong to E-Income exhibit higher profitability, dividend payout and positive stock returns.

\subsubsection{E-IPO vs. ATHEX}

Panel $\mathrm{G}$ of Table 1 shows that firms that belong to E-IPO are significantly different compared to the constituent firms of ATHEX. Therefore, $H_{0} 4.1$ can be rejected. As expected, firms that form E-IPO tend to exhibit higher growth measures (MVBV) and price to earnings ratio (PE). They also are more profitable (NPM) and exhibit higher leverage (DEBTE). This implies that along with using equity finance following their flotation on the ASE, they also use borrowings to finance their expansion and growth. To appear more attractive and improve their financial picture, the specific firms distribute higher dividends (DIVSH) to shareholders and pay their creditors (CREDT) timely. They also display lower debtor turnover (DEBT), which signifies that they extend their credit policy to attract more customers and influence their profitability positively. Panel G shows that due to the fact that firms that belong to E-IPO are new-listed on the ASE, their stock returns display higher volatility (VOLAT). The K-W test (Panel E of Table 2) shows that firms that belong to E-IPO are larger, and exhibit higher profitability, liquidity, leverage, dividend payout and volatility in stock returns.

\subsubsection{FTSE 20 vs. E-Income}

Panel $\mathrm{H}$ of Table 1 shows that firms that belong to FTSE 20 display significantly different financial characteristics compared to firms that form E-Income. ${ }^{7}$ Thus, $H_{04.1}$ can be rejected. Panel $\mathrm{H}$ indicates that constituent firms of FTSE 20 tend to be larger (SALESHA) and exhibit higher leverage (TLSFU). In contrast, firms that belong to E-Income are more profitable (EPS) and display higher growth potential (MVBV). The results show that between firms that belong to FTSE 20 and firms that belong to E-Income, the latter experience higher stock returns (AR), implying that the stock market attributes higher value to firms with higher profitability and future prospects. The results of the K-W test (Panel F of Table 2) are similar with those of the logistic regression presented above.

\subsubsection{FTSE International vs. E-Income}

Panel I of Table 1 shows that firms that belong to FTSE International are significantly different compared to the constituent firms of E-Income. Thus, $H_{0} 4.1$ can be rejected. The results show that firms that belong to FTSE International are larger (SALETAS) and exhibit higher holdings to total assets (HOLTA). To meet their business objectives and support their corporate strategy domestically and internationally, the specific firms tend to use higher debt capital (DEBTE). Their higher leverage appears to adversely affect their profitability (EPS), liquidity (CFSH) and dividend payout (DIVSH). The international exposure and the higher related risk of the firms that belong to FTSE 
International, in association with their lower profitability, appear to introduce volatility in their stock returns (VOLAT). The K-W test (Panel G of Table 2) shows that firms that belong to FTSE International are larger, and exhibit lower profitability, higher leverage and volatility in stock returns.

\subsubsection{Size And Firms' Financial Attributes}

Panel $\mathrm{J}$ of Table 1 shows that large firms exhibit significantly different financial attributes compared to small firms. Thus, $H_{05}$ can be rejected. The categorization of the sample firms is performed using the market value (MV). The dependent dummy variable in the logistic regression takes 1 for large firms, and 0 for small firms.

The accounting policy choice and actions of large firms are highly visible and subjected to criticism and judgment by various financial and non-financial interested parties, including trade unions, environmental and government authorities, community representatives, etc. Therefore, large firms are inclined to build a solid financial and business picture to avoid political costs, and meet the needs and expectations of the interested parties. Panel $\mathbf{J}$ shows that large firms exhibit lower growth measures (MVBV), implying that, ceteris paribus, the rate of marginal growth for a large firm would tend to be lower compared to a small firm that is at the beninning of its operation. Failing to meet their financial obligations and abide by the underlying debt covenants would have severe implications for large firms. Although large firms display higher leverage measures (DEBTE), their profitability is not affected negatively. In contrast, they exhibit higher profitability (ROCE), dividend payout (DIVSH) and liquidity (CUR). As expected, large firms exhibit higher holdings to total assets (HOLTA), while they display lower debtor turnover (DEBT). This implies that the sales policy of large firms is such that they sell on credit to attract more customers and increase their revenues. The lower debtor turnover does not appear to adversely affect their creditor turnover (CREDT), which is found to be higher for large firms. The financial figures of large firms that are presented above constitute an overall favourable financial picture, which seems to be appreciated by the stock market as it appears by the positive coefficient of stock returns (AR). The K-W test (Panel $\mathrm{H}$ of Table 2) shows that large firms exhibit higher profitability, liquidity, leverage, holdings to total assets, dividend payout and positive stock returns.

\subsubsection{Growth And Firms' Financial Attributes}

Panel K of Table 1 shows that firms with high growth prospects are significantly different compared to firms with low growth measures. Thus, $H_{05}$ can be rejected. The categorization of the sample firms is performed using the variable "market value to book value" (MVBV) (see Collins and Kothari, 1989). The dependent dummy variable in the logistic regression takes 1 for high growth firms, and 0 for low growth firms.

Following the higher uncertainty that characterizes their future prospects, high growth firms experience higher volatility in stock returns (VOLAT). They also are smaller (NAVSH and SALETAS), implying that, under certain circumstances, a small firm is more likely to grow larger in the future than a firm that is already large. Panel $\mathrm{K}$ shows that high growth firms have higher intangible assets (INTTA), including investments, development projects, etc., that are likely to generate returns in future periods. This potential is also reflected by the higher price to earnings ratio (PE) that high growth firms exhibit. Despite their higher leverage measures (TLSFU and DEBTE), the specific set of firms appears to be more profitable (NPM, ROCE and EPS), and also pay higher dividends (DIVSH). The higher debtor turnover (DEBT) shows that high growth firms collect debts quickly, in order to reinforce their operations and liquidity, and eventually their growth potential. Likewise, the K-W test (Panel I of Table 2) shows that high growth firms are smaller, and exhibit higher profitability, liquidity, leverage, dividend payout and volatility in stock returns.

\subsubsection{Profitability And Firms' Financial Attributes}

Panel L of Table 1 shows that firms with high profitability exhibit significantly different financial attributes compared to firms with low profitability. Thus, $H_{0}$ can be rejected. The categorization of the sample firms is performed using the earnings per share (EPS). The dependent dummy variable in the logistic regression takes 1 for high profitability firms, and 0 for low profitability firms. 
High profitability firms tend to be larger (SALESHA) and exhibit higher holdings to total assets (HOLTA). They also exhibit higher growth measures (MVBV) and price to earnings ratio (PE). Following their higher profitability, the specific firms distribute higher dividends (DIVSH) to their shareholders. High profitability firms display higher debtor turnover (DEBT), which allows them to pay their creditors timely (CREDT) and positively affect their liquidity (CFM). Panel L shows that the higher leverage (IGEAR) that they exhibit does not appear to impact negatively on their financial performance. In fact, high profitability firms are able to adequately meet their interest expenses as it is shown by the positive coefficient of interest cover (INTCOV). The higher profitability together with the favourable financial figures and growth potential of the specific set of firms appear to have a positive impact on their stock returns (AR). The $\mathrm{K}-\mathrm{W}$ test (Panel J of Table 2) shows that high profitability firms display higher size, growth, liquidity, leverage, dividend payout and positive stock returns.

\subsubsection{Liquidity And Firms' Financial Attributes}

Panel $\mathrm{M}$ of Table 1 shows that firms with high liquidity are significantly different compared to firms with low liquidity. Hence, $H_{05}$ can be rejected. The categorization of the sample firms is performed using the current ratio (CUR). The dependent dummy variable in the logistic regression takes 1 for high liquidity firms, and 0 for low liquidity firms.

The results show that high liquidity firms are larger (SALETAS) and more profitable (NPM and ROSC). They also exhibit higher debtor (DEBT), creditor (CREDT) and stock (STOCKT) turnover. This shows that high liquidity firms sell their stock and collect debts quickly, and thus, they are able to pay their creditors timely. This would tend to increase their reliability and reputation, and further strengthen their creditability. High liquidity firms also exhibit higher leverage (CGEAR), which does not appear to adversely affect their profitability. In fact, their higher profitability, allows them to meet their interest obligations, and thus, display higher interest cover (INTCOV). The favourable financial performance of high liquidity firms that is described above together with the higher dividend (DIVCOV) that they pay to shareholders would tend to improve their financial profile and positively influence their stock returns (AR). The K-W test (Panel K of Table 2) shows that high liquidity firms are larger, and display higher profitability, dividend payout and price to earnings ratio. In contrast to the results of the logistic regression presented above, the specific firms appear to exhibit lower leverage measures.

\subsubsection{Leverage And Firms' Financial Attributes}

Panel $\mathrm{N}$ of Table 1 shows that firms with high leverage exhibit significantly different financial attributes compared to firms with low leverage. Thus, $H_{05}$ can be rejected. The categorization of the sample firms is performed using the variable "debt to equity" (DEBTE). The dependent dummy variable in the logistic regression takes 1 for high leverage firms, and 0 for low leverage firms.

High leverage firms appear to be larger (SALESHA) and exhibit higher growth prospects (MVBV) and price to earnings ratio (PE). The latter attributes show that the specific firms use more debt capital in order to facilitate and support their growth potential. Following their higher level of borrowing, high leverage firms display lower profitability (NPM, ROCE and EPS). To enhance their liquidity (CFM) and their ability to faithfully meet their financial obligations, high leverage firms collect debts quickly (DEBT), and at the same time, they exhibit higher stock turnover (STOCKT). This implies that money is tied up for less time in stocks, and thus, firms get to make profit on the stock quicker, which would in turn reinforce their competitive position and liquidity. Despite their lower profitability, high leverage firms appear to retain a smaller amount of profits (PLOWB), in order to pay higher dividends (DIVCOV and DIVYI), and thus, attract more investors and obtain more equity finance. The K-W test (Panel L of Table 2) shows that high leverage firms are larger, while they appear to exhibit lower liquidity.

\subsection{Dividend Distribution And Firms' Financial Attributes}

Panel $\mathrm{O}$ of Table 1 shows that firms that distribute dividends are significantly different compared to firms that pay no dividends to shareholders. Thus, $H_{06}$ can be rejected. The results indicate that firms that pay dividends tend to be larger (NAVSH), and as expected, they exhibit higher profitability measures (OPM, NPM and EPS). They 
also display higher leverage (DEBTE), which does not affect negatively their profitability or dividend policy. Firms may in fact distribute dividends in order to impress the market participants, and give positive signals to stakeholders, such as lenders and financial institutions, about their financial position and prospects. The higher leverage that they exhibit and the financial obligations that result do not appear to have a negative impact on their liquidity. Panel $\mathrm{O}$ shows that firms that pay dividends display higher liquidity (CFM), which is reinforced by the higher debtor turnover (DEBT) that they exhibit. The specific firms also display higher price to earnings ratio (PE), which implies that they possibly operate in a growth area, and thus, they pay dividends to attract investors and satisfy the market's expectations and perceptions. The results of the K-W test (Panel M of Table 2) are similar with those of the logistic regression presented above, and also show that firms that pay dividends display higher growth measures and positive stock returns.

\subsection{Trading Volume And Firms' Financial Attributes}

Panel $\mathrm{P}$ of Table 1 shows that firms that display a positive change in trading volume have distinctive financial characteristics compared to firms with negative change in trading volume. Therefore, $H_{0} 7$ can be rejected. The results show that investors are attracted by firms that are credible and exhibit strong financial performance and promising future prospects. In particular, firms with positive change in trading volume tend to exhibit higher growth measures (MVBV) and appear to be more profitable (ROSC). The specific firms display higher liquidity (CUR), and also are able to pay their creditors (CREDT) timely. They display a positive change in trading volume, implying that they attract investors' interest, also because they exhibit lower leverage (DEBTE). Lower leverage signifies that firms will incur lower interest expenses, and that debt covenants will possibly be less restrictive. The specific firms also display higher dividend payout (DIVSH and DIVCOV), which would tend to be appreciated by investors. Overall, the results indicate that firms with positive change in trading volume exhibit a favourable financial picture, which appears to satisfy investors and lead to positive stock returns (AR). The results of the K-W test (Panel N of Table 2) are similar with those of the logistic regression presented above, and also show that firms that display a positive change in trading volume appear to be larger.

6

Conclusions

This study describes the financial characteristics of firms listed on the ASE in association with their stock return behaviour. It is evident that high volatility in stock returns is negatively associated with stock returns. It appears that in order to positively influence their stock returns, firms need not just maximize their profits or minimize their costs. They also need to identify and meet market participants' needs. In particular, they need to provide assurance that they abide by the regulation and that their accounting statements reflect their true and fair view and assist in the development of correct predictions about firms' future financial performance. High liquidity and dividend payout, and significant growth prospects accompanied by solid profitability appear to positively impact on stock returns. On the other hand, uncertainty about firms' future performance would introduce volatility in stock returns.

With regard to the ASE indices, firms that belong to the primary ASE index, i.e. ATHEX, overall display higher size, leverage, profitability and liquidity. It appears that in comparison with firms outside ATHEX, the specific firms are subjected to more intensive scrutiny and inspection. Thus, they tend to structure their decision-making and policy choice so as to improve their financial picture and positively influence investors' perceptions. Firms that are inside a stock market index appear to be more careful and tend to take seriously into account financial analysts' forecasts and investors' expectations. It appears that the financial attributes and actions of firms that belong to market indices are highly associated with the categorization criteria and objectives of each respective index. For example, the actions and choices of new-listed firms that form E-IPO aim to strengthen their financial position as well as attract more investors and impress the market participants. In general, firms that attract investors' interest and exhibit high trading volume tend to display higher growth, profitability and dividend payout.

The study has assessed listed firms' financial performance based on size, growth, profitability, liquidity and leverage. It appears that firms tend to display lower retained earnings and pay higher dividends, even if they exhibit relatively higher leverage and lower profits, in order to attract investors. In a similar vein, firms tend to pay their creditors timely and show higher creditor turnover in order to satisfy the creditors as well as other potential lenders 
and market participants. This happens especially when firms have high leverage. Firms that are politically visible, either in terms of size or profitability, tend to exhibit high awareness in their actions and decisions to avoid political costs or attracting the attention of authorities. Firms with high growth tend to display high leverage to support their expansion. Firms, especially those with high profitability, appear to take into account earnings forecasts and structure their accounting policy choices accordingly, in order not to make investors sceptical about their managerial abilities and future prospects.

This study is useful for investors, financial analysts, accounting regulators and stock market authorities. It gives significant insight about listed firms' financial profile and covers major aspects of their business and financial performance. The study enhances the understanding of regulators and government authorities about listed firms' financial characteristics and needs, which would enable them to improve the current accounting regulation, reinforce the current auditing and supervisory framework, and better protect investors' interests. In that respect, the study gives significant insight to investors about the behaviour of stock returns and the factors that influence their fluctuation, which should be considered when investors set up their portfolios and investment strategy. The study, therefore, contributes to the improvement of the current market setting aiming at the attraction of foreign capital, the reflection of the true and fair firm financial picture on stock returns, and the immediate and correct reaction of stock prices to the announcement of financial events. Categorising the sample firms based on key accounting measures, the study provides a scanning of the Athens stock market. This provides a break-down of listed firms' financial position, attributes and behaviour, and can be the basis for the reinforcement of the efficiency of the stock market, the reduction of earnings manipulation, and the making of efficient decisions. Overall, the study provides the setting that users of accounting information could use to describe and interpret the stock return behaviour in association with firm financial numbers and changes.

\section{Notes}

${ }^{1}$ Following that shareholders generally prefer a stable income stream to volatile earnings (Gordon, 1964), firms may resort to hedging in order to reduce the adverse impact of earnings volatility on their accounting numbers and financial position, stock returns and compensation (Fama, 1980; Ndubizu and Tsetsekos, 1992).

${ }^{2}$ It is evident that stock returns are more sensitive to financial analysts' forecasts of firm financial performance than to changes in accounting numbers themselves (Chung and Jo, 1996; La Porta, 1996). According to Imhoff (1992), financial analysts attribute higher value to firms with more predictable earnings, smaller forecast revisions, lower probability of bad news announcements, stable performance and profitability measures over time, and whose accounting numbers meet the respective forecasts.

${ }^{3}$ In an efficient stock market, the stock returns should faithfully and reliably reflect the firms' actions and decisions. In this case, the stock returns would depict the true and fair view of the firm. The examination of the association between the financial attributes of listed firms and the sign of the stock returns is closely related to how efficient the Athens stock market is, and how well market participants see through firms' judgement and operations.

${ }^{4}$ FTSE 20 and FTSE International have been designed by the ASE in collaboration with the London Stock Exchange and FTSE International Limited.

${ }^{5}$ To categorise firms into those with high and low volatility in stock returns, the analysis has also been performed using the coefficient of variation, i.e. standard deviation divided by the mean of annual stock returns. The results (not presented here) appear to be similar with those presented in the paper.

${ }^{6}$ The study has also used the Pearson correlation test to capture the relationship between stock volatility and stock returns. The results (not presented here) show that the volatility in stock returns is negatively correlated with stock returns.

${ }^{7}$ The comparison between firms that belong to FTSE 20 and ATHEX (not presented here) shows that the former tend to exhibit higher size (SALESHA), leverage (DEBTE) and profitability (NPM). They also display higher stock returns (AR). 


\section{Acknowledgements}

The authors gratefully acknowledge the financial support provided by the Research Project Archimedes: "Innovative Financial Instruments, Portfolio Management and Growth Potential of the Hellenic Stock Market". This paper is co-funded by the European Social Fund and National Resources- EPEAEK II.

\section{References}

1. Aboody, D. and R. Kaznik (2000), CEO Stock Option Awards and the Timing of Corporate Voluntary Disclosures, Journal of Accounting and Economics, 29 (1): 73-100.

2. Adiel, R. (1996), Reinsurance and the Management of Regulatory Ratios and Taxes in the Property-Casualty Insurance Industry, Journal of Accounting and Economics, 22: 207-240.

3. Ali, A. and K. Kumar (1994), The Magnitudes of Financial Statement Effects and Accounting Choice; The Case of the Adoption of SFAS 87, Journal of Accounting and Economics, 18: 89-114.

4. Alli, K., A. Khan and G. Ramirez (1993), Determinants of Corporate Dividend Policy: A factorial Analysis, Financial Review, 28: 523-547.

5. Balsam, S., I. M. Haw and S. B. Lilien (1995), Mandated Accounting Changes and Managerial Discretion, Journal of Accounting and Economics, 20: 3-29.

6. Bartov, E., D. Givoly and C. Hayn (2002), The Rewards to Meeting or Beating Earnings Expectations, Journal of Accounting and Economics, 33: 173-204.

7. Beaver, W. (2002), Perspectives on Recent Capital Market Research, Accounting Review, 77: 453-474.

8. Bekaert, G. and G. Wu (2000), Asymmetric Volatility and Risk in Equity Markets, Review of Financial Studies, 13: 1-42.

9. Blacconiere, W. G., R. M. Bowen, S. E. Sefcik and C. H. Stinson (1991), Determinants of the Use of Regulatory Accounting Principles by Savings and Loans, Journal of Accounting and Economics, 14: 167201.

10. Blacconiere, W. G. and D. M. Patton (1994), Environmental Disclosures, Regulatory Costs and Changes in Firm Value, Journal of Accounting and Economics, 18: 357-377.

11. Brennan, M. and S. Titman (1994), A Study of Monthly Fund Returns and Performance Evaluation Techniques, Journal of Financial and Quantitative Analysis, 29 (3): 419-444.

12. Brous, P. and O. Kini (1994), The Valuation Effects of Equity Issues and the Level of Institutional Ownership: Evidence from Analysts' Earnings Forecasts, Financial Management, 23: 33-46.

13. Brown, L. and M. Caylor (2005), A Temporal Analysis of Quarterly Earnings Thresholds: Propensities and Valuation Consequences, Accounting Review, (April).

14. Brown, L. and H. Higgins (2005), Managers' Forecast Guidance of Analysts: International Evidence, Journal of Accounting and Public Policy, 24 (4): 280-299.

15. Burgstahler, D. and I. Dichev (1997), Earnings Management to Avoid Earnings Decreases and Losses, Journal of Accounting and Economics, 24: 99-126.

16. Bushee, B. (2001), Do Institutional Investors Prefer Near-Term Earnings over Long-Run Value?, Contemporary Accounting Research, 18: 207-246.

17. Cahan, S. (1992), The Effect of Antitrust Investigations on Discretionary Accruals: A Refined Test of the Political Cost Hypothesis, The Accounting Review, (January): 77-95.

18. Cahan, S., B. Chavis and R. Elmendorf (1997), Earnings Management of Chemical Firms in response to Political Costs from Environmental Legislation, Journal of Accounting, Auditing and Finance, (Winter): $37-$ 65.

19. Campbell, J. and L. Hentschel (1992), No News is Good News, Journal of Financial Economics, 31: 281318.

20. Christie, A. (1982), The Stochastic Behaviour of Common Stock Variances: Value, Leverage, and Interest Rate Effects, Journal of Financial Economics, 10: 407-432.

21. Christie, A. A. and J. L. Zimmerman (1994), Efficient and Opportunistic Choices of Accounting Procedures: Corporate Control Contests, The Accounting Review, 69: 539-566.

22. Chung, K. and H. Jo (1996), The Impact of Security Analysts' Monitoring and Marketing Functions on the Market Value of Firms, Journal of Financial and Quantitative Analysis, 31: 493-512. 
23. Chung, R., M. Firth and J. Kim (2002), Institutional Monitoring and Opportunistic Earnings Management, Journal of Corporate Finance, 8 (1): 29-48.

24. Collins, D. and S. Kothari (1989), An Analysis of the Intertemporal and Cross-Sectional Determinants of Earnings Response Coefficients, Journal of Accounting and Economics, 11: 143-181.

25. D' Souza, J. M. (1998), Rate-regulated Enterprises and Mandated Accounting Changes: The Case of Electric Utilities and Post-retirement Benefits Other than Pensions (SFAS No. 106), The Accounting Review, 73: 387410.

26. Daniel, K. and S. Titman (1997), Evidence on the Characteristics on Cross-Sectional Variation in Stock Returns, Journal of Finance, 52: 1-33.

27. Datar, V., N. Naik and R. Radcliffe (1998), Liquidity and Stock Returns: An Alternative Test, Journal of Financial Markets, 1: 203-219.

28. Dechow, P. and R. Sloan (1991), Executive Incentives and the Horizon Problem: An Empirical Investigation, Journal of Accounting and Economics, 14: 51-89.

29. Dechow, P., R. Sloan and A. Sweeney (1995), Detecting Earnings Management, The Accounting Review, (April): 193-225.

30. Dechow, P., R. Sloan and A. Sweeney (1996), Causes and Consequences of Earnings Manipulation: An Analysis of Firms Subject to Enforcement Actions by the SEC, Contemporary Accounting Research, (Spring): 1-36.

31. Dechow, P., J. Sabino and R. Sloan (1997), Implications of Non-discretionary Accruals for Earnings Management and Market-based Research, Working Paper, University of Michigan.

32. DeFond, M. L. and C. W. Park (1997), Smoothing Income in Anticipation of Future Earnings, Journal of Accounting and Economics, 23: 115-139.

33. Dempsey, S., G. Laber and M. Rozeff (1993), Dividend Policies in Practice: Is There an Industry Effect?, Quarterly Journal of Business and Economics, 32: 3-13.

34. Dey, M. (2005), Turnover and Return in Global Stock Markets, Emerging Markets Review, 6 (1): $45-67$.

35. Domnowitz, I., J. Glen and A. Madhavan (2001), Liquidity, Volatility, and Equity Trading Costs Across Countries, and Over Time, International Finance, 4: 221-255.

36. Doukas, J., P. McNight and C. Pantzalis (2005), Security Analysis, Agency Costs and UK Firm Characteristics, International Review of Financial Analysis, 14: 493-507.

37. Duke, J. and H. Hunt (1990), An Empirical Examination of Debt Covenant Restrictions and AccountingRelated Debt Proxies, Journal of Accounting and Economics, 12: 45-63.

38. Dye, R. and R. Verrecchia (1995), Discretion vs. Uniformity: Choices Among GAAP, The Accounting Review, 70: 389-415.

39. Easley, G., S. Hvidkjaer and M. O’Hara (2002), Is Information Risk a Determinant of Asset Returns, Journal of Finance, 58(5): 2185-2221.

40. Eberhart, A. and A. Siddique (2002), The Long-Term Performance of Corporate Bonds (and Stocks) following Seasoned Equity Offerings, Review of Financial Studies, 15: 1385-1406.

41. Eldenburg, L. and N. Soderstrom (1996), Accounting System Management by Hospitals Operating in a Changing Regulatory Environment, The Accounting Review, 71: 23-42.

42. Elliott, R. and P. Jacobson (1994), Commentary: Costs and Benefits of Business Information Disclosure, Accounting Horizons, 8/4: 80-96.

43. Erickson, M. and S. W. Wang (1999), Earnings Management by Acquiring Firms in Stock for Stock Mergers, Journal of Accounting and Economics, 27: 149-176.

44. Fama, E. (1980), Agency Problems and the Theory of the Firm, Journal of Political Economy, (April): 288307.

45. Fields, T., T. Lys and L. Vincent (2001), Empirical Research on Accounting Choice, Journal of Accounting and Economics, 31 (1-3): 255-307.

46. Francis, J., D. Hanna and L. Vincent (1996), Causes and Effects of Discretionary Asset Write-offs, Journal of Accounting Research, 34 (Supplement): 117-134.

47. Francis, J. (2001), Discussion of Empirical Research on Accounting Choice, Journal of Accounting and Economics, 31 (1-3): 309-319.

48. French, K., G. Schwert and R. Stambaugh (1987), Expected Stock Returns and Volatility, Journal of Financial Economics, 19: 3-29. 
49. Gaver, J. and K. Gaver (1998), The Relation between Nonrecurring Accounting Transactions and CEO Cash Compensation, The Accounting Review, 73: 235-253.

50. Gordon, M. (1964), Postulates, Principles and Research in Accounting, The Accounting Review (April): 251263.

51. Guidry, F., A. J. Leone and S. Rock (1999), Earnings-based Bonus Plans and Earnings Management by Business-unit Managers, Journal of Accounting and Economics, 26: 113-142.

52. Han, J. C. Y. and S. W. Wang (1998), Political Costs and Earnings Management of Oil Companies During the 1990 Persian Gulf Crisis, The Accounting Review, 73: 103-117.

53. Healy, P. (1985), The Impact of Bonus Schemes on the Selection of Accounting Principles, Journal of Accounting and Economics, 7: 85-107.

54. Hirst, D. E. and P. E. Hopkins (1998), Comprehensive Income Reporting and Analysts' Valuation Judgements, Journal of Accounting Research, 36 (Supplement): 47-75.

55. Holthausen, R. W. (1990), Accounting Method Choice: Opportunistic Behaviour, Efficient Contracting and Information Perspectives, Journal of Accounting and Economics, 12: 207-218.

56. Holthausen, R. and R. Watts (2001), The Relevance of the Value-Relevance Literature for Financial Accounting Standard Setting, Journal of Accounting and Economics, 31: 3-75.

57. Imhoff, E. (1992), The Relation Between Perceived Accounting Quality and Economic Characteristics of the Firm, Journal of Accounting and Public Policy, 11: 97-118.

58. Jensen, M. C. and W. H. Meckling (1976), Theory of the Firm: Managerial Behaviour, Agency Costs and Ownership Structure, Journal of Financial Economics, 3: 305-360.

59. Jensen, M. (1986), Agency Costs of Free Cash Flow, Corporate Finance and Takeovers, American Economic Review, 76: 323-329.

60. Jensen, G., D. Solberg and T. Zorn (1992), Simultaneous Determination of Insider Ownership, Debt, and Dividend Policies, Journal of Financial and Quantitative Analysis, 27: 247-263.

61. Karmon, D. and C. Lubwama (1997), An Events-study Approach to Detecting Income-smoothing Activities: Some Evidence from Multinational Corporations, Journal of International Accounting, Auditing \& Taxation, 6 (1): 75-95.

62. Kasznik, R. (1999), On the Association Between Voluntary Disclosure and Earnings Management, Journal of Accounting Research, 37: 57-81.

63. Kim, M. S. and W. Kross (1998), The Impact of the 1989 Change in Bank Capital Standards on Loan Loss Provisions and Loan Write-offs, Journal of Accounting and Economics, 25: 69-99.

64. Kim, C., J. Morley and C. Nelson (2004), Is There a Positive Relationship Between Stock Market Volatility and the Equity Premium?, Journal of Money, Credit, and Banking, 36: 339-360.

65. Kothari, S. (2001), Capital Market Research in Accounting, Journal of Accounting and Economics, 31: 105231.

66. La Porta, R. (1996), Expectations and the Cross-Section of Stock Returns, Journal of Finance, 51 (5): 17151742.

67. Lambert, R. (2001), Contracting Theory and Accounting, Journal of Accounting and Economics, 32 (1-3): 387.

68. Lamont, O. (1997), Cash Flow and Investment: Evidence from Internal Capital Markets, Journal of Finance, 52: 83-109.

69. Lesmond, D., J. Ogden and C. Trzinka (1999), A New Estimate of Transactions Costs, Review of Financial Studies, 12: 1113-1141.

70. Levitt, A. (1998), The Importance of High Quality Accounting Standards, Accounting Horizons, 12: 79-82.

71. Lewellen, W. G., T. Park and B. T. Ro (1996), Self-serving Behaviour in Managers' Discretionary Information Disclosure Decisions, Journal of Accounting and Economics, 21: 227-251.

72. Lundstrum, L. (2003), Firm Value, Information Problems and the Internal Capital Market, Review of Quantitative Finance and Accounting, 21: 141-156.

73. Matsumoto, D. (2002), Management's Incentive to Avoid Negative Earnings Surprises, Accounting Review, 77: 483-514.

74. Matsunaga, S. (1995), The Effects of Financial Reporting Costs on the Use of Employee Stock Options, The Accounting Review, 70: 1-26. 
75. May, D. (1995), Do Managerial Motives Influence Firm Risk Reduction Strategies? Journal of Finance, 50: 1291-1308.

76. Merton, R. (1974), On the Pricing of Corporate Debt: The risk Structure of Interest Rates, Journal of Finance, 29: 449-470.

77. Moses, D. (1987), Income Smoothing and Incentives: Empirical Test Using Accounting Changes, The Accounting Review, (April): 358-373.

78. Myers, S. and N. Majluf (1984), Corporate Financing And Investment Decisions When Firms Have Information that Investors Do Not Have, Journal of Financial Economics, 13: 187-221.

79. Ndubizu, G. and G. Tsetsekos (1992), Functional Currency Smoothing and Managerial Incentives: An Empirical Test, The International Journal of Accounting, 27: 27-37.

80. Nohel, T. and V. Tarhan (1998), Share Repurchases and Firm Performance: New Evidence on the Agency Costs of Free Cash Flow, Journal of Financial Economics, 49: 187-222.

81. Noronha, G., D. Shome and G. Morgan (1996), The Monitoring Rationale for Dividends and the Interaction of Capital Structure and Dividend Decisions, Journal of Banking and Finance, 20: 439-454.

82. Perry, S. and T. Williams (1994), Earnings Management Preceding Management Buyout Offers, Journal of Accounting and Economics, 18: 157-179.

83. Pope, P., S. Young and K. Peasnell (2000), Accruals Management to Meet Earnings Targets: Did Cadbury Make a Difference?, British Accounting Review, 32: 415-445.

84. Press, E. and J. Weintrop (1990), Accounting-Based Constraints in Public and Private Debt Agreements, Journal of Accounting and Economics, 12: 65-95.

85. Rajan, R., H. Servaes and L. Zingales (2000), The Cost of Diversity: The Diversification Discount and Inefficient Investment, Journal of Finance, 55: 35- 80.

86. Richardson, S., S. Teoh and P. Wysocki (2004), The Walkdown to Beatable Analyst Forecasts: The Role of Equity Issuance and Insider Trading Incentives, Contemporary Accounting Research, 21 (4).

87. Rouwenhorst, K. (1999), Local Return Factors and Turnover in Emerging Stock Markets, Journal of Finance, 54: 1439-1464.

88. Rozeff, M. (1982), Growth, Beta and Agency Costs as Determinants of Dividend Payout Ratios, Journal of Financial Research, 5: 249-259.

89. Sang-Gyung, J., A. Marathe and H. Shawky (2003), Liquidity and Stock Markets in Emerging Equity Markets, Emerging Markets Review, 4: 1-24.

90. Scott, W. (1997), Financial Accounting Theory, Prentice Hall Inc.

91. Shin, H. and R. Stulz (1998), Are Internal Capital Markets Efficient?, Quarterly Journal of Economics, 113: 531-552.

92. Skinner, D. and R. Sloan (2002), Earnings Surprises, Growth Expectations, and Stock Returns: Don't Let an Earnings Torpedo Sink Your Portfolio, Review of Accounting Studies, 7: 289-312.

93. Strong, N. (1992), Modelling Abnormal Returns: A Review Article, Journal of Business Finance and Accounting, 19 (4): 533-553.

94. Stulz, R. (1990), Managerial Discretion and Optimal Financing Policies, Journal of Financial Economics, 26: $3-27$.

95. Subramanyam, K. R. (1996), The Pricing of Discretionary Accruals, Journal of Accounting and Economics, 22: 249-281.

96. Sweeney, A. P. (1994), Debt-Covenant Violations and Managers' Accounting Responses, Journal of Accounting and Economics, 17: 281-308.

97. Watts, R. and J. Zimmerman (1986), Positive Theory of Accounting, Prentice-Hall, Englewood-Cliffs, NJ.

98. Watts, R. and J. Zimmerman (1990), Positive Accounting Theory: A Ten Year Perspective, The Accounting Review, (January): 131-156.

99. Wermers, R. (2000), Mutual Fund Performance: An Empirical Decomposition into Stock Picking Talent, Style, Transactions Costs, and Expenses, Journal of Finance, 55: 1655-1695.

100. Yermack, D. (1997), Good Timing: CEO Stock Option Awards and Company News Announcements, Journal of Finance, 52: 449-476.

101. Young, S. (1998), The Determinants of Managerial Accounting Policy Choice: Further Evidence for the UK, Accounting and Business Research, 28 (2): 133-143.

102. Zimmerman, J. (1983), Taxes and Firm Size, Journal of Accounting and Economics, 5: 119-149. 
Appendix 1 Sample industrial sectors

\begin{tabular}{|l|c|}
\hline \multicolumn{1}{|c|}{ Industry } & No of Firms \\
\hline Chemicals & 11 \\
\hline Construction and building materials & 39 \\
\hline Industrial goods and services & 25 \\
\hline Food and beverage & 34 \\
\hline Retail & 16 \\
\hline Health care & 7 \\
\hline Basic resources & 17 \\
\hline Travel and leisure & 18 \\
\hline Media and entertainment & 15 \\
\hline Oil and gas & 4 \\
\hline Personal care and household products & 41 \\
\hline Technology & \\
\hline Telecommunications & 22 \\
\hline Utilities & \\
\hline Total & \\
\hline
\end{tabular}

\begin{tabular}{|c|c|}
\hline \multicolumn{2}{|c|}{ Appendix 2 Accounting measures used as explanatory variables } \\
\hline Size & \\
\hline MV & Market value \\
\hline $\mathrm{BV}$ & Book value \\
\hline SALESHA & Sales per share \\
\hline NAVSH & Net asset value per share \\
\hline SALETAS & Sales to total assets \\
\hline \multicolumn{2}{|l|}{ Growth } \\
\hline MVBV & Market value to book value \\
\hline \multicolumn{2}{|l|}{ Profitability } \\
\hline OPM & Operating profit margin \\
\hline NPM & Net profit margin \\
\hline ROSC & Return on shareholders' capital \\
\hline ROCE & Return on capital employed \\
\hline EPS & Earnings per share \\
\hline \multicolumn{2}{|l|}{ Liquidity } \\
\hline CREDT & Creditor turnover \\
\hline DEBT & Debtor turnover \\
\hline CUR & Current ratio \\
\hline CASH & Cash ratio \\
\hline QUI & Quick (acid) ratio \\
\hline CFM & Cash flow margin \\
\hline $\mathrm{CFSH}$ & Cash flow per share \\
\hline \multicolumn{2}{|l|}{ Leverage } \\
\hline TLSFU & Total liabilities to shareholders' funds \\
\hline CGEAR & Capital gearing \\
\hline INTCOV & Interest cover \\
\hline IGEAR & Income gearing \\
\hline DEBTE & Debt to equity \\
\hline \multicolumn{2}{|c|}{ Other variables } \\
\hline INTTA & Intangibles to total assets \\
\hline VOLAT & Volatility in annual stock returns \\
\hline DIVSH & Dividend per share \\
\hline DIVYI & Dividend yield \\
\hline DIVCOV & Dividend cover \\
\hline PLOWB & Plowback (retention) ratio \\
\hline $\mathrm{PE}$ & Price to earnings ratio \\
\hline
\end{tabular}




\section{NOTES}

